\title{
Leading-edge receptivity for bodies with mean aerodynamic loading
}

\author{
By P. W. HAMMERTON ${ }^{1}$ AND E. J. KERSCHEN ${ }^{2}$ \\ ${ }^{1}$ School of Mathematics, University of East Anglia, Norwich NR4 7TJ, UK \\ ${ }^{2}$ Department of Aerospace \& Mechanical Engineering, University of Arizona, Tucson, AZ 85721, USA
}

(Received 12 May 1998 and in revised form 1 December 2004)

Boundary-layer receptivity in the leading-edge region of a cambered thin airfoil is analysed for the case of a low-Mach-number flow. Acoustic free-stream disturbances are considered. Asymptotic results based on large Reynolds number $\left(U^{2} / \omega v \gg 1\right)$ are presented, supplemented by numerical solutions. The influence of mean aerodynamic loading enters the theory through a parameter $\mu$, which provides a measure of the flow speed variations in the leading-edge region, due to flow around the leading edge from the lower surface to the upper. A Strouhal number based on airfoil nose radius, $S=\omega r_{n} / U$, also enters the theory. The variation of the receptivity level as a function of $\mu$ and $S$ is analysed. Modest levels of aerodynamic loading are found to decrease the receptivity level for the upper surface of the airfoil, while the receptivity is increased for the lower surface. For larger angles of attack close to the critical angle for boundary layer separation, a local rise in the receptivity occurs for the upper surface, while on the lower surface the receptivity decreases. These effects are more pronounced at larger values of $S$. While the Tollmien-Schlichting wave does not emerge until a downstream distance of $O\left(\left(U^{2} / \omega v\right)^{1 / 3} U / \omega\right)$, the amplitude of the Tollmien-Schlichting wave is influenced by the acoustic free-stream disturbances only in a relatively small region near the leading edge, of length approximately $4 U / \omega$. The numerical receptivity coefficients calculated, together with the asymptotic eigenfunctions presented, provide all the necessary information for transition analysis from the interaction of acoustic disturbances with leading-edge geometry.

\section{Introduction}

The transition of a boundary-layer flow from laminar to turbulent is strongly influenced by free-stream disturbances. The process by which free-stream disturbances generate instability waves in the boundary layer is known as receptivity (Morkovin 1969). Energy is transferred from long-wavelength disturbances in the free stream to the much shorter wavelength Tollmien-Schlichting waves in the boundary layer. This transfer requires a wavelength conversion mechanism, which is usually produced by non-parallel mean flow effects due to short-scale streamwise variations in the mean flow. These streamwise gradients may arise from viscous boundary-layer growth associated with the leading-edge region (Goldstein 1983; Hammerton \& Kerschen 1996, 1997), or from localized regions farther downstream in the boundary layer, such as regions with sudden changes in surface geometry (Ruban 1984; Goldstein 1985; Choudhari \& Kerschen 1990) or marginally separated regions (Goldstein, Leib \& Cowley 1987). Additional background information on receptivity can be found in 
Goldstein \& Hultgren (1989), Kerschen (1990), Kozlov \& Ryzhov (1990) and Saric, Reed \& Kerschen (2002).

Leading-edge receptivity was first considered for the Blasius boundary layer on a flat plate (Goldstein 1983). A high-Reynolds-number asymptotic analysis was formulated for an incompressible two-dimensional flow of free-stream speed $U$, together with a small-amplitude time-harmonic perturbation of frequency $\omega$, equivalent to the incompressible limit of an acoustic wave propagating parallel to the mean flow. Two streamwise regions enter the analysis, one region of $O(U / \omega)$, where the inviscid pressure field and slip velocity induced by the free-stream disturbance drives the unsteady motion in the boundary layer, and a second region farther downstream at distance $O\left(\left(v \omega / U^{2}\right)^{-1 / 3} U / \omega\right)$, where the disturbance is governed by the tripledeck structure, corresponding to the high-Reynolds-number asymptotic form of the Orr-Sommerfeld equation in the vicinity of the lower branch.

In the first region of Goldstein's analysis where the streamwise length scale is $U / \omega$, the unsteady disturbance in the boundary layer is governed by the linearized unsteady boundary layer equation (LUBLE). Far downstream in the LUBLE region, the solution consists of a Stokes wave, and a set of asymptotic eigenfunctions that contain velocity but not pressure fluctuations. These asymptotic eigenfunctions are equivalent to those obtained by Lam \& Rott $(1960,1993)$ and Ackerberg \& Phillips (1972). The asymptotic analysis for distances far downstream determines the form of the eigenfunctions, but not their coefficients $C_{i}$.

The wavelengths of the asymptotic eigenfunctions shorten progressively with distance downstream. Eventually, the self-induced pressure field associated with the displacement thickness of each asymptotic eigenfunction becomes significant, and the triple-deck structure replaces the LUBLE as the correct asymptotic approximation to the Navier-Stokes equation. The first asymptotic eigenfunction of the LUBLE matches onto the Tollmien-Schlichting (TS) wave solution of this triple-deck region. Thus, the form of the free-stream disturbance and the geometry close to the nose influence the amplitude of the TS wave only through the coefficient $C_{1}$ of the first asymptotic eigenfunction. For this reason, $C_{1}$ is known as the 'receptivity coefficient'. The numerical value of the receptivity coefficient cannot be determined by asymptotic methods. Instead it must be extracted from numerical solutions of the LUBLE, by comparison with the asymptotic form far downstream in the LUBLE region. This was accomplished for the flat-plate case by Goldstein, Sockol \& Sanz (1983) and Heinrich \& Kerschen (1989).

The analysis of Goldstein for a flat plate was the first theoretical description of the fundamental mechanisms of leading-edge receptivity. However, aerodynamic bodies designed for subsonic flow generally have finite thickness distributions with a parabolic leading edge. Hammerton \& Kerschen $(1996,1997)$ considered a thin symmetric airfoil at zero angle of attack, with a plane acoustic wave incident at arbitrary angle, and examined the influence of the leading-edge geometry on receptivity. The structure of the development of the instability remains similar to that for a flat-plate; the asymptotic eigenfunctions of the LUBLE are modifications of the Lam-Rott eigenfunctions, which take account of the effects of mean pressure gradient and surface curvature. Attention was focused on the variation of the receptivity level with the nose radius of the airfoil and the incidence angle $\theta$ of the acoustic field. The nose radius $r_{n}$ of the airfoil enters the analysis as a Strouhal number, $S=\omega r_{n} / U$. The receptivity decreases rapidly as $S$ is increased, dropping to less than half the flat-plate level when $S=0.3$. For low Mach numbers, the receptivity to oblique acoustic waves is much stronger than that for an acoustic wave propagating parallel to the airfoil 
chord. In wind tunnel tests, reflections of acoustic waves from the sidewalls can lead to oblique impingment on the leading edge, increasing receptivity levels significantly (see Kerschen 1990 and Saric \& White 1998). Inclusion of the leading-edge thickness effect goes some way towards modelling experimental investigations (Saric et al. 1995; Saric \& White 1998) and allowing comparisons with related numerical simulations (Reed \& Lyttle 1998; Haddad \& Corke 1998; Erturk \& Corke 2001).

In this paper, the receptivity for a cambered airfoil at an angle of attack to a mean flow is considered in the high-Reynolds-number limit $\left(\epsilon^{6}=\nu \omega / U_{e}^{2} ; \epsilon \ll 1\right)$. The physics of the receptivity process for this realistic geometry is considerably more intricate than that for previous studies of leading-edge receptivity. The previous studies were restricted to symmetric mean flows, involving zero-pressure-gradient boundary layers or favourable pressure gradients that decayed monotonically to zero with downstream distance. In contrast, for the present geometry the favourable pressure gradient at the stagnation point is followed by an even stronger favourable pressure gradient as the flow travels around the leading edge. The pressure gradient on the upper surface then becomes adverse and the boundary layer may tend toward separation, leading to a minimum in the wall shear stress which is followed by a relatively slow recovery with downstream distance as the adverse pressure gradient relaxes. Pressure gradients are known to exert a strong influence on the development of instability waves, and it is natural to anticipate equally strong effects on the leading-edge receptivity process.

In our analysis, attached boundary-layer flow is assumed so that the wall shear stress is everywhere positive and finite. This assumption is consistent with aerodynamic design for cruise conditions. At high angles of attack near the boundary of the aerodynamic envelope, some airfoils may develop a small region of 'marginal separation' just downstream of the leading edge, in which the wall shear stress nearly vanishes. Although we consider situations involving adverse pressure gradients and small values of wall shear stress, we do not approach the critical angle of attack corresponding to marginal separation. Thus the marginal separation receptivity mechanism analysed by Goldstein et al. (1987) is not relevant to the present study.

The structure of the paper is as follows. In $\S 2$ a high-Reynolds-number asymptotic analysis is formulated for an incompressible two-dimensional flow about a thin cambered airfoil. In $\S 2.1$, the steady slip velocity on the airfoil surface is determined using thin-airfoil theory, followed in $\S 2.2$ by the derivation of the equation governing the unsteady boundary-layer flow in terms of suitable coordinates. Assuming that the unsteady perturbations in the free stream are small, and letting $\epsilon \rightarrow 0$, the unsteady disturbances in the boundary layer are governed by the LUBLE.

The solution in the LUBLE region is analysed in $\S 3$. When considering the subsequent development of instabilities in the boundary-layer flow, the asymptotic form of the unsteady solution far downstream in the LUBLE region is required. The derivation of the asymptotic form of both the steady and unsteady components of the solution is a necessary part of the presentation, but mathematical details are given in the Appendices instead of the body of the paper in order to avoid interrupting the description of the evolution of the boundary-layer disturbance.

In $\S 4$, results are presented for the inviscid pressure field and slip velocity produced by the interaction of a free-stream acoustic wave with an airfoil. The unsteady slip velocity in the vicinity of the leading edge depends on the incidence angle of the acoustic wave in a way that is fundamentally different for small and large values of the reduced acoustic frequency $k=\omega b / c$, where $b$ is the airfoil semi-chord and $c$ is the speed of sound. In $\S 5$, numerical solutions of the steady boundary layer equation and the LUBLE are obtained, and compared with the asymptotic eigenfunctions of $\S 3$. 


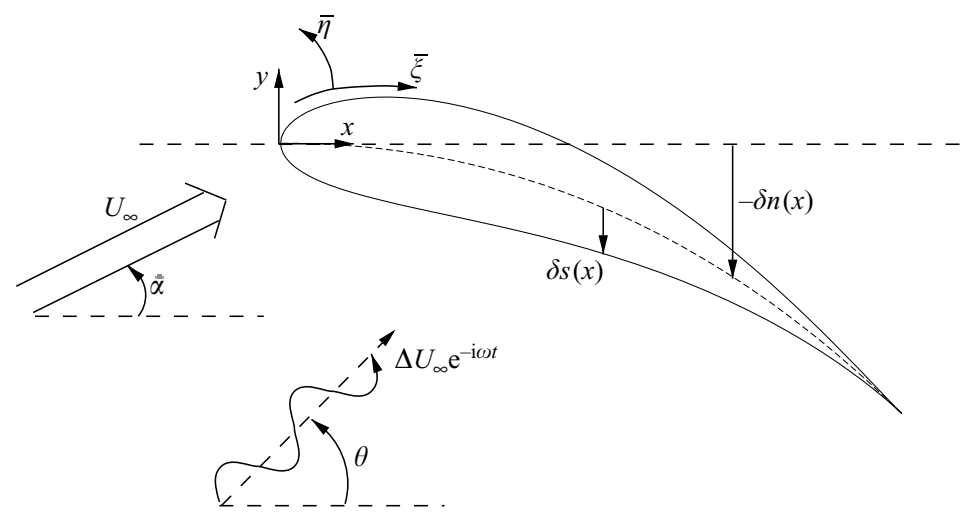

FIGURE 1. An illustration of the physical situation of interest: a thin cambered airfoil of chord $2 b$ is at angle $\widehat{\alpha}$ to a uniform flow of speed $U_{\infty}$, with a plane acoustic wave incident at an angle $\theta$.

This allows determination of the receptivity coefficient as a function of leading-edge geometry, airfoil shape and angle of attack, and the characteristics of the free-stream acoustic wave.

\section{Formulation}

We consider a thin cambered airfoil of chord $2 b$ at an angle of attack $\widehat{\alpha}$ to a uniform flow of speed $U_{\infty}$. A plane acoustic wave of frequency $\omega$, propagating at an angle $\theta$, is assumed to be incident on the airfoil as illustrated in figure 1. Two-dimensional low-Mach-number flow is considered. Since the Mach number is small, the mean flow can be analysed using incompressible theory. For the unsteady component of the flow, most features of interest can also be analysed using incompressible theory. The influence of compressibility on the unsteady component of the flow is discussed in $\S 4$. Since the Reynolds number is assumed large, the flow field is inviscid and irrotational everywhere except in the vicinity of the airfoil surface. The behaviour of the flow in the boundary layer adjacent to the airfoil surface depends crucially on the pressure gradient imposed by the outer inviscid flow. Therefore, relevant features of the inviscid mean flow are presented in $\S 2.1$. The equations governing the boundary-layer flow are formulated in $\S 2.2$.

\subsection{Inviscid mean flow}

We introduce Cartesian coordinates $(x, y)$ normalized by the airfoil semi-chord $b$, with the origin located at the airfoil leading edge and the $x$ - and $y$-coordinate axes parallel and normal to the airfoil chord line at the leading edge, as illustrated in figure 1. This particular choice of coordinate system proves convenient for the present analysis, in which attention is focused on the leading-edge region. The camber and thickness of the airfoil are assumed of $O(\delta)$ where $\delta \ll 1$. Explicitly factoring out the scaling parameter $\delta$, the airfoil surface is defined by

$$
y=\delta(-n(x) \pm s(x)), \quad 0 \leqslant x \leqslant 2,
$$

where the \pm sign applies on the upper and lower surface, respectively, and the camber and thickness functions $n(x)$ and $s(x)$ are defined in figure 1. The angle of attack $\widehat{\alpha}$, measured with respect to the axes defined above, is also assumed to be small (to avoid separation) and to be comparable to the thickness and camber; hence we write $\widehat{\alpha}=\delta \alpha$. 
The inviscid mean flow past the airfoil can then be calculated using thin-airfoil theory. Specifically, the no-penetration boundary condition on the upper and lower surfaces of the airfoil can be linearized and transferred to $y=0^{ \pm}$, respectively. The mean-flow perturbation due to the presence of the airfoil is found to have the $y$-components

$$
U_{\infty} \delta\left(-\alpha+n^{\prime}(x) \pm s^{\prime}(x)\right) \quad \text { on } \quad 0 \leqslant x \leqslant 2, \quad y=0^{ \pm},
$$

where $n^{\prime}(x)$ and $s^{\prime}(x)$ denote the derivatives of these functions with respect to $x$. The mean slip velocity on the airfoil surface, $U_{s}$, immediately follows (Cheng $\&$ Rott 1954),

$$
\frac{U_{s}(x)}{U_{\infty}}=1-\frac{\delta}{\pi}\left(\int_{0}^{2} \frac{s^{\prime}\left(x_{1}\right)}{x_{1}-x} \mathrm{~d} x_{1}+\left[\frac{2-x}{x}\right]^{1 / 2} \int_{0}^{2}\left[\frac{x_{1}}{2-x_{1}}\right]^{1 / 2} \frac{n^{\prime}\left(x_{1}\right)-\alpha}{x_{1}-x} \mathrm{~d} x_{1}\right)+O\left(\delta^{2}\right) .
$$

A circulatory component has been included in (2.3), its magnitude fixed by the Kutta condition at the trailing edge.

The thin-airfoil expansion (2.3) becomes non-uniform in the vicinity of the leading edge, and must be supplemented by a local expansion. For an airfoil with a parabolic leading edge of (dimensional) radius $r_{n}$, the small-argument expansions of the camber and thickness distribution have the form

$$
n(x)=O\left(x^{2}\right), \quad s(x)=s_{1} x^{1 / 2}[1+O(x)],
$$

where $s_{1}=\left(2 r_{n} / \delta^{2} b\right)^{1 / 2}$. Following the approach of Hammerton \& Kerschen (1996, henceforth denoted HK1), parabolic coordinates scaled on the airfoil nose radius $r_{n}$ are introduced,

$$
x+\mathrm{i} y=\frac{1}{2} \frac{r_{n}}{b}\left[(\bar{\xi}+\mathrm{i} \bar{\eta})^{2}+1\right] .
$$

At leading order in the local coordinates, the airfoil surface is defined by the parabola $\bar{\eta}=1$. The inviscid flow in the vicinity of the parabolic nose can be obtained in the form of a complex potential by means of conformal mappings, giving a slip velocity

$$
U_{s}(\bar{\xi})=U_{e}\left(\frac{\bar{\xi}}{\left(\bar{\xi}^{2}+1\right)^{1 / 2}}+\mu \frac{1}{\left(\bar{\xi}^{2}+1\right)^{1 / 2}}\right) .
$$

The first term of (2.6) corresponds to symmetric flow past the nose, while the second term is an antisymmetric flow around the nose, from the lower surface to the upper, when the parameter $\mu$ is positive. The parameters $U_{e}$ and $\mu$ in (2.6) are determined by matching with the thin-airfoil expression (2.3). Comparing the small- $x$ behaviour of (2.3) and the large- $\bar{\xi}$ behaviour of (2.6), we obtain

$$
\begin{aligned}
\frac{U_{e}}{U_{\infty}} & =1+\frac{\delta}{\pi}\left(\frac{s_{1}}{\sqrt{2}}-\int_{0}^{2}\left[\frac{\mathrm{d} s}{\mathrm{~d} x_{1}}-\frac{s_{1}}{2 x_{1}^{1 / 2}}\right] \frac{\mathrm{d} x_{1}}{x_{1}}\right), \\
\mu & =\frac{2 \delta b^{1 / 2}}{r_{n}^{1 / 2}}\left(\alpha-\frac{1}{\pi} \int_{0}^{2} \frac{n^{\prime}(x)}{\left[x_{1}\left(2-x_{1}\right)\right]^{1 / 2}} \mathrm{~d} x_{1}\right) .
\end{aligned}
$$

The effective free-stream speed near the leading edge, $U_{e}$, is influenced by the full thickness distribution of the airfoil. The aerodynamic loading parameter for the leading-edge region, $\mu$, depends on the airfoil nose radius and semi-chord, as well as the angle of attack and the full camber distribution of the airfoil. Since $r_{n}=O\left(\delta^{2} b\right)$, $\mu$ is $O(1)$. From (2.6), the stagnation point on the lower surface is given by $\bar{\xi}=-\mu$. 


\subsection{Boundary-layer flow}

The boundary-layer flow in the vicinity of the leading edge is also analysed most conveniently in parabolic coordinates. For the mean boundary layer, the nose radius $r_{n}$ remains the appropriate length scale in the streamwise direction. However, for the unsteady flow, a second streamwise length scale, $U_{e} / \omega$, is also relevant. Specifically, Goldstein's (1983) analysis of leading-edge receptivity for the flat-plate boundary layer showed that the receptivity process takes place in a region where the distance from the leading edge is $O\left(U_{e} / \omega\right)$. To examine the boundary-layer flow in this region, we introduce new coordinates,

$$
\xi=S^{1 / 2} \bar{\xi}, \quad \eta=\epsilon^{-3} S^{1 / 2}(\bar{\eta}-1)
$$

where

$$
S=\frac{\omega r_{n}}{U_{e}}, \quad \epsilon=\left(\frac{\nu \omega}{U_{e}^{2}}\right)^{1 / 6} .
$$

The parameter $S$ is a Strouhal number based on the airfoil nose radius, while $\epsilon^{6}$ is the reciprocal of the Reynolds number based on the disturbance length scale $U_{e} / \omega$. The analysis presented here considers the small- $\epsilon$ limit but with $S=O(1)$. Note that $\epsilon^{-3} S^{1 / 2}=\left(U_{e} r_{n} / \nu\right)^{1 / 2}$, so that $\eta$ is the normal coordinate of conventional boundary-layer theory.

The non-dimensional vorticity equation, expressed in terms of the streamfunction $\Psi$ (which has been normalized by $\epsilon^{3} U_{e}^{2} / \omega$ ), then becomes

$$
\begin{array}{r}
\Psi_{\eta \eta t}+\frac{\partial\left(\Psi_{\eta \eta} / H^{2}, \Psi\right)}{\partial(\xi, \eta)}-\frac{\Psi_{\eta \eta \eta \eta}}{H^{2}}=\epsilon^{6}\left(\frac{\Psi_{\eta \eta \xi \xi}}{H^{2}}+\left[\frac{\Psi_{\eta \eta}}{H^{2}}\right]_{\xi \xi}-\frac{\partial\left(\Psi_{\xi \xi} / H^{2}, \Psi\right)}{\partial(\xi, \eta)}-\Psi_{\xi \xi t}\right) \\
+\epsilon^{12}\left[\frac{\Psi_{\xi \xi}}{H^{2}}\right]_{\xi \xi}, \quad(2.11)
\end{array}
$$

where $H=\left(\xi^{2}+S\right)^{1 / 2}$ and the time $t$ has been non-dimensionalized by $1 / \omega$. Equation (2.11) is exact except for approximation of the metric coefficient by $H$ (see HK1). The viscous flow satisfies the no-slip boundary conditions on the body surface,

$$
\Psi=\Psi_{\eta}=0 \quad \text { on } \quad \eta=0,
$$

while at the outer edge of the boundary layer, the viscous flow matches to the inviscid slip velocity (non-dimensionalized by $U_{e}$ ),

$$
H^{-1} \Psi_{\eta} \rightarrow \widetilde{U}_{s}(\xi, t) \quad \text { as } \quad \eta \rightarrow \infty
$$

where $\widetilde{U}_{s}(\xi, t)$ contains both the steady and unsteady components of the slip velocity, see (3.4).

For $O(1)$ values of $\xi$, the terms on the right-hand side of (2.11) can be neglected, leading to the unsteady boundary-layer equation. The unsteady component of the flow, a small perturbation to the mean flow, then satisfies the linearized unsteady boundarylayer equation (LUBLE). It is shown in $\S 3$ that the solution of the LUBLE contains components whose wavelengths progressively shorten with distance downstream. Thus, for the unsteady component of the flow, terms on the right-hand side of (2.11) become significant when $\xi=O\left(\epsilon^{-1}\right)$, and the correct asymptotic approximation to (2.11) then takes on the triple-deck structure. The asymptotic matching of these two streamwise regions is discussed briefly at the end of $\S 3$. 


\section{Analysis of receptivity region}

In this section, we consider the region of the boundary layer where the receptivity takes place. Since the streamwise length scale for this region is $U_{e} / \omega$, the boundary layer is governed by the form of (2.11) corresponding to the limit $\epsilon \rightarrow 0, \xi=O(1)$. Subsequently, the behaviour of this solution at large values of $\xi$ will be considered, in anticipation of matching with the Orr-Sommerfeld region that exists farther downstream. For $\xi=O(1)$ and $\epsilon \rightarrow 0$, the terms on the right-hand side of (2.11) can be ignored. Integrating once with respect to $\eta$ then gives the unsteady boundary-layer equation,

$$
H^{2} \Psi_{\eta t}+\left(\Psi_{\eta} \Psi_{\xi \eta}-\Psi_{\eta \eta} \Psi_{\xi}\right)-\xi H^{-2} \Psi_{\eta}^{2}-\Psi_{\eta \eta \eta}=-H^{2} P_{\xi},
$$

where the pressure gradient $P_{\xi}(\xi, t)$ is obtained by matching to the outer (inviscid) flow,

$$
P_{\xi}(\xi, t)=-H \frac{\partial \widetilde{U}_{s}}{\partial t}-\widetilde{U}_{s} \frac{\partial \widetilde{U}_{s}}{\partial \xi} .
$$

Up to this point our analysis has paralleled that of HK1, utilizing nearly the same notation. For the symmetric mean flow case considered in HK1, the unsteady boundary-layer region was analysed using the streamwise coordinate $\xi$ (which is based on the length scale $U_{e} / \omega$ ). This allowed easy comparison with the flat-plate analysis of Goldstein (1983). However, for the non-zero angle of attack case considered here, no corresponding flat-plate analysis is possible because the boundary layer would separate at the leading edge. In addition, the presence of airfoil thickness, camber and angle of attack significantly complicates the algebra. The analysis of the mean flow is most naturally carried out in terms of a streamwise coordinate based on the nose radius $r_{n}$. Noting the parabolic nature of the governing equations, one can see that the mean-flow stagnation point is the natural origin for the streamwise coordinate. Thus we introduce the new streamwise coordinate,

$$
w=\bar{\xi}+\mu=S^{-1 / 2} \xi+\mu,
$$

where $\mu$ is the angle-of-attack parameter defined in (2.8). The mean-flow stagnation point is now given by $w=0$, the nose corresponds to $w=\mu$, and $w$ is scaled on the nose radius $r_{n}$ rather than the disturbance length scale $U_{e} / \omega$.

Because the mean flow is expressed most simply in terms of the streamwise coordinate $w$, it turns out that $w$ is also the most convenient coordinate for analysis of the unsteady flow. Our analysis assumes $S=O(1)$, so that $w=O(1)$ also corresponds to $\xi=O(1)$. Later in this section we analyse the behaviour of the solution for large $\xi$, in order to examine the development of the Lam-Rott asymptotic eigenfunctions $\psi_{i}$, and the eventual evolution of the first eigenfunction $\psi_{1}$ into the TS wave when $\xi=O(1 / \epsilon)$. This large- $\xi$ analysis will be presented in terms of large $w$ for algebraic simplicity, but we emphasize that it is the quantity $S^{1 / 2} w$ which is required to be large.

For a cambered airfoil at non-zero angle of attack, the slip velocity is given in terms of the new coordinate $w$ by

$$
\widetilde{U}_{s}(w, t)=\frac{w}{h}+\Delta u_{s}(w) \mathrm{e}^{-\mathrm{i} t},
$$

where $h^{2}=1+(w-\mu)^{2}$. Here the steady contribution $U_{s}=w / h$ follows from (2.6), while the time-dependent component $u_{s}(w)$ depends on the particular form of the free-stream disturbance, as discussed in $\S 4$. Since we are concerned only with small-amplitude free-stream disturbances, that is $\Delta \ll 1$, the steady and unsteady components of the flow field can be analysed separately. Thus, the streamfunction 
within the boundary layer can be written in the corresponding form

$$
\Psi(w, \eta)=S^{1 / 2} w \phi(w, \eta)+\Delta \psi(w, \eta) \mathrm{e}^{-\mathrm{i} t},
$$

where the factor $S^{1 / 2} w$ has been extracted from the mean component of the streamfunction in order to simplify the matching condition as $\eta \rightarrow \infty$. The steady boundary-layer flow then satisfies

$$
\phi_{\eta \eta \eta}+\phi_{\eta \eta} \phi+w\left(\phi_{\eta \eta} \phi_{w}-\phi_{\eta} \phi_{\eta w}\right)-\beta\left(\phi_{\eta}^{2}-1\right)=0,
$$

where the pressure gradient parameter $\beta(w ; \mu)$ is given by

$$
\beta=\frac{1-\mu w+\mu^{2}}{h^{2}},
$$

together with boundary conditions

$$
\phi=\phi_{\eta}=0 \text { at } \eta=0 \text { and } \quad \phi_{\eta} \rightarrow 1 \quad \text { as } \quad \eta \rightarrow \infty .
$$

The time-dependent component of the boundary-layer flow satisfies the linearized unsteady boundary-layer equation (LUBLE),

$$
\left.\begin{array}{c}
\mathscr{F}(\psi)=S^{1 / 2} h^{2} \frac{\mathrm{d} p}{\mathrm{~d} w}, \\
\mathscr{F}(\psi) \equiv \psi_{\eta \eta \eta}+\left(\phi+w \phi_{w}\right) \psi_{\eta \eta}+\left(\mathrm{i} S h^{2}+\frac{w^{2}-\mu^{2}-1}{h^{2}} \phi_{\eta}-w \phi_{\eta w}\right) \psi_{\eta} \\
+w\left(\phi_{\eta \eta} \psi_{w}-\phi_{\eta} \psi_{\eta w}\right), \\
\frac{\mathrm{d} p}{\mathrm{~d} w}=\left(\mathrm{i} S h-\frac{1-\mu(w-\mu)}{h^{3}}\right) u_{s}-\frac{w}{h} \frac{\mathrm{d} u_{s}}{\mathrm{~d} w},
\end{array}\right\}
$$

with boundary conditions

$$
\psi=\psi_{\eta}=0 \quad \text { at } \quad \eta=0 \quad \text { and } \quad \psi_{\eta} \rightarrow S^{1 / 2} h u_{s} \quad \text { as } \quad \eta \rightarrow \infty .
$$

These equations must be solved numerically for each value of $S$ and $\mu$, and for each different free-stream disturbance, although various simplifications arise as will be seen later. The numerical solutions are described in $\S 5$. However, as discussed in $\S 1$, we are primarily interested in the solution of these equations in the large- $\xi$ (or large- $w$ ) limit, where a component of the solution to the LUBLE matches onto the TS wave solution of the Orr-Sommerfeld equation. In the next two subsections, we develop large- $w$ asymptotic expansions for $\phi(w, \eta)$ and $\psi(w, \eta)$.

\subsection{Large-w expansion of the steady boundary-layer equation}

In order to determine the form of the asymptotic eigenfunction of the LUBLE that matches onto the TS wave far downstream, the large- $w$ expansion of the mean flow is required. The large- $w$ expansion of the pressure gradient parameter is

$$
\beta \sim-\frac{\mu}{w}+\frac{\left(1-\mu^{2}\right)}{w^{2}}+\frac{\mu\left(3-\mu^{2}\right)}{w^{3}}+O\left(\frac{1}{w^{4}}\right) .
$$

The expansion of $\beta$ must be carried out up to $O\left(w^{-3}\right)$ in order to determine the exponential and $O(1)$ algebraic dependence of the asymptotic eigenfunction of the LUBLE, as is shown in Appendix B. For $\mu>0$, an adverse pressure gradient arises on the upper surface $(w>0)$. This adverse pressure gradient decays to zero much more slowly than the favourable pressure gradient arising in the symmetric-mean-flow case $(\mu=0)$. 
Guided by the $\mu=0$ case (Van Dyke 1964), we find that the asymptotic expansion of the mean flow far downstream is given by

$$
\phi(w, \eta) \sim F(\eta)+\frac{p_{1}(\eta)}{w}+\frac{p_{2}(\eta) \log w^{2}}{w^{2}}+\frac{p_{3}(\eta)}{w^{2}}+\frac{p_{4}(\eta) \log w^{2}}{w^{3}}+\frac{p_{5}(\eta)}{w^{3}}+O\left(\frac{1}{w^{\gamma_{2}}}\right)
$$

where the fractional power $\gamma_{2}=3.774$ arises as an eigensolution (Libby \& Fox 1963). Here $F(\eta)$ is the Blasius solution and the $p_{i}(\eta)$ are determined in Appendix A. Note that the parameter $\mu$ appears in the $p_{i}$. As for the symmetric-mean-flow case described in HK1, the large- $w$ asymptotic form (3.12) of the mean flow is not fully determined by the local conditions far downstream (see Appendix A). The functions $p_{3}(\eta)$ and $p_{5}(\eta)$ contain a coefficient $B_{1}(\mu)$ that must be determined by comparing the asymptotic form (3.12) with a numerical solution obtained by integrating (3.6) from the stagnation point $w=0$.

The behaviour of the mean flow close to the wall is required in the analysis for the asymptotic eigenfunction of the LUBLE. This is given by (Appendix A)

$$
\phi \sim \frac{\eta^{2} U_{0}^{\prime}(w)}{2}+\frac{\eta^{3} U_{0}^{\prime \prime}(w)}{6}-\frac{\eta^{5} F_{0}^{\prime \prime 2}}{5 !}+O\left(\frac{\eta^{2}}{w^{\gamma_{2}}}, \frac{\eta^{5}}{w}\right)
$$

where $U_{0}^{\prime}(w)$ and $U_{0}^{\prime \prime}(w)=-\beta(w)$ are the wall shear stress and the profile curvature at the wall, given by the asymptotic expansions (A 7) and (3.11) respectively, accurate to $O\left(w^{-3}\right)$. We also have $F_{0}^{\prime \prime}=F^{\prime \prime}(0)$.

\subsection{Large- $S^{1 / 2} w$ expansion of the linearized unsteady boundary-layer equation}

We next consider the evolution of the unsteady component of the flow, which is governed by (3.9). As in the symmetric-mean-flow case considered in HK1, far downstream in the LUBLE region $\left(S^{1 / 2} w \gg 1\right)$ the unsteady component of the flow consists of a particular solution, $\psi_{p}$, determined entirely by the local conditions far downstream, together with a set of asymptotic eigenfunctions,

$$
\psi(w, \eta ; S, \mu)=\psi_{p}(w, \eta ; S, \mu)+\sum_{i} C_{i}(S, \mu) \psi_{i}(w, \eta ; S, \mu) .
$$

The particular solution is a generalization of the classical Stokes layer solution, driven by the local value of the unsteady pressure gradient (3.9). The asymptotic eigenfunctions $\psi_{i}$ are generalizations of the Lam-Rott eigenfunctions (Lam \& Rott 1960, 1993; Ackerberg \& Phillips 1972), taking account of the non-Blasius mean flow. (A second set of asymptotic eigenfunctions for the LUBLE were derived by Brown \& Stewartson (1973), but their relationship to TS wave development has not been established.)

The eigenfunctions are 'asymptotic' because they exist only for $S^{1 / 2} w \gg 1$, where the mean flow takes on a slowly varying character. The $\psi_{i}$ depend on the meanflow characteristics far downstream, but are independent of the unsteady freestream disturbance in the downstream region. The coefficients $C_{i}$ of the asymptotic eigenfunctions are determined by the characteristics of the unsteady free-stream disturbance and the mean boundary-layer flow in the region nearer the leading edge where $S^{1 / 2} w=O(1)$. One of the asymptotic eigenfunctions, which we label $\psi_{1}$, matches onto the growing TS wave in the Orr-Sommerfeld region farther downstream, where $S^{1 / 2} w=O(1 / \epsilon)$. It is only through the receptivity coefficient $C_{1}$ that the unsteady free-stream disturbance influences the amplitude of the TS wave. In the remainder 
of this paper, most attention is focused on this particular eigenfunction and on its coefficient $C_{1}$.

The form of the general asymptotic eigenfunction $\psi_{i}$ is obtained in Appendix B. The asymptotic eigenfunctions have a two-layer structure (Ackerberg \& Phillips 1972), with a new inner layer of width $\eta=O\left(S^{-1 / 2} w^{-1}\right)$. The leading-order expression for the general eigenfunction $\psi_{i}$ is given by

$$
\psi_{i}^{(0)}= \begin{cases}F_{0}^{\prime \prime}\left(\frac{S w^{2}}{2}\right)^{\tau_{i}} \exp \left(T_{i}^{(0)}(w)\right) h_{i}(n), & n=S^{1 / 2} w \eta=O(1), \\ S^{1 / 2} w\left(\frac{S w^{2}}{2}\right)^{\tau_{i}} \exp \left(T_{i}^{(0)}(w)\right) F^{\prime}(\eta), & \eta=O(1)\end{cases}
$$

where $\tau_{i}$ and $T_{i}^{(0)}(w)$ are given by (B 20) and (B 21) respectively, and

$$
h_{i}(n)=\frac{\int_{0}^{n}(n-\widetilde{n}) \operatorname{Ai}\left(\mathrm{e}^{\mathrm{i} \pi / 4} \rho_{i}^{-1 / 2} \widetilde{n}-\rho_{i}\right) \mathrm{d} \widetilde{n}}{\int_{0}^{\infty} \operatorname{Ai}\left(\mathrm{e}^{\mathrm{i} \pi / 4} \rho_{i}^{-1 / 2} \widetilde{n}-\rho_{i}\right) \mathrm{d} \widetilde{n}}
$$

which is obtained from (B 24) using $M=n\left[1+O\left(w^{-1}\right)\right]$. Here $\rho_{i}$ is the $i$ th root of $\operatorname{Ai}^{\prime}(-\rho)=0$. The leading term of $T_{i}^{(0)}(w)$ is proportional to $(\mathrm{i}-1) w^{3}$, so that the asymptotic eigenfunctions exhibit exponential decay and a gradually shortening wavelength as they propagate downstream.

In the next section, numerical solutions for the LUBLE are compared to the asymptotic eigenfunction $\psi_{1}$ in order to extract values of the receptivity coefficient $C_{1}$. The two most convenient points of comparison are the unsteady component of the wall shear, $\psi_{\eta \eta}(\eta=0)$, and the unsteady component of the boundary-layer thickness which is related to the value of the streamfunction far from the wall, $\psi(\eta \rightarrow \infty)$. For the first eigensolution,

$$
\begin{aligned}
& \psi_{1}^{\prime \prime}(\eta=0) \sim 0.4356(1+\mathrm{i})\left(\frac{S w^{2}}{2}\right)^{\tau_{1}+1} \exp \left(T_{1}^{(0)}(w)\right)\left[1+O\left(w^{-\gamma_{2}+3}\right)\right], \\
& \psi_{1}(\eta \rightarrow \infty) \sim S^{1 / 2} w\left(\frac{S w^{2}}{2}\right)^{\tau_{1}} \exp \left(T_{1}^{(0)}(w)\right)\left[1+O\left(w^{-\gamma_{2}+3}\right)\right],
\end{aligned}
$$

with $T_{1}^{(0)}(w)$ given by (B 21) with $\rho=\rho_{1}$ and

$$
\tau_{1}=-0.6921+1.9878\left(1-6.182 \mu^{2}\right) S i .
$$

The exponent for the first neglected term in (3.17) and (3.18) is $-\gamma_{2}+3=-0.774$. This term is followed by a sequence of closely spaced correction terms of $O\left(w^{-1} \ln ^{2} w\right)$, $O\left(w^{-1} \ln w\right)$ and $O\left(w^{-1}\right)$, as can be seen from the analysis presented in Appendix B.

Before proceeding to numerical solutions of (3.9), we first consider the validity of the LUBLE. While the eigenfunctions obtained here are uniformly valid solutions for the LUBLE as $S^{1 / 2} w \rightarrow \infty$, they are not uniformly valid solutions of the full equations governing the development of the viscous flow at large distances downstream. The wavelengths of the eigenfunctions decrease with distance downstream, increasing the importance of terms involving streamwise derivatives. When $S^{1 / 2} w=O\left(\epsilon^{-1}\right)$, terms on the right-hand side of (2.11), which were neglected in forming the LUBLE, now become significant. An irrotational layer outside the mean boundary layer, driven by 
the oscillating displacement thickness of the eigenfunction, must then be considered, and the pressure gradient imposed by the motion in this outer layer appears in the leading-order equations governing the wall layer. This coupled viscous-inviscid interaction has the triple-deck structure, corresponding to the small- $\epsilon$ asymptotic approximation to the Orr-Sommerfeld equation in the vicinity of the lower branch. A complete treatment of the linear development of the instability wave would require an asymptotic solution for the TS wave of the Orr-Sommerfeld equation, taking account of the mean pressure-gradient distribution on the airfoil. However, for $O(1)$ values of $S$, with $\epsilon$ and $\delta$ small quantities of the same order, the Orr-Sommerfeld region is influenced by the full airfoil camber and thickness distributions, necessitating a general development that does not seem justified in the present context.

In this paper we restrict ourselves to examining only the receptivity process, and the remainder of the paper is concerned with determining the receptivity coefficient $C_{1}$ as a function of the nose radius parameter $S$, the aerodynamic loading parameter $\mu$, and the characteristics of the free-stream disturbance. This is accomplished by comparing numerical solutions with the asymptotic solutions obtained above.

\section{Free-stream disturbances}

In order to calculate the unsteady flow in the boundary layer, the inviscid flow field which drives the unsteady motion in the boundary layer must be determined. In this section we consider the inviscid flow field produced by the interaction of a free-stream acoustic wave with the airfoil. The acoustic wave is assumed to be incident on the airfoil at an angle $\theta$ with respect to the airfoil chord, as illustrated in figure 1. The slip velocity and surface pressure fields generated by this interaction drive the unsteady motion in the boundary layer, leading to the generation of a TS wave. Since the outer inviscid flow is irrotational, the unsteady pressure field is easily related to the unsteady velocity field. Thus, we present results for the unsteady slip velocity in the leading-edge region.

For a low-Mach-number flow, the acoustic wavelength $2 \pi c / \omega$ is long compared to the hydrodynamic length scale $U_{e} / \omega$. Thus, outside the boundary layer, the unsteady flow in the vicinity of the leading edge is incompressible and irrotational. Potential flow theory then shows that this local flow has the form

$$
u_{s}(w)=\kappa_{s}(\theta) \frac{w-\mu}{h}+\kappa_{a}(\theta) \frac{1}{S^{1 / 2} h}
$$

where $h^{2}=1+(w-\mu)^{2}$ as before. The functions multiplying $\kappa_{s}$ and $\kappa_{a}$ are $O(1)$ in the region of receptivity $\left(S^{1 / 2} w=O(1)\right)$. Noting that $w-\mu=\bar{\xi}$, the parabolic coordinate with origin at the leading edge defined by $(2.5)$, it is seen that $(w-\mu) / h$ and $1 / h$ correspond to purely symmetric and antisymmetric flow about the leading edge, respectively. The two components of (4.1) exhibit quite different behaviour. The symmetric component is zero at the nose $(w=\mu)$, and approaches one for large $w$. In contrast, the antisymmetric component has its maximum value at the nose, and approaches zero at large $w$. The coefficients $\kappa_{s}$ and $\kappa_{a}$ multiplying these eigenfunctions are independent of the nose geometry, but depend on the free-stream disturbance, being determined by global features of the unsteady flow.

The nature of the unsteady interaction of the acoustic wave with the airfoil depends upon the magnitude of the acoustic reduced frequency $k=\omega b / c$, where $c$ is the speed of sound in the undisturbed medium. For $k \ll 1$, the acoustic wavelength is long compared to the airfoil chord and classical unsteady airfoil theory for incompressible 
flow gives (see HK1)

$$
\kappa_{s}=\cos \theta, \quad \kappa_{a}=\left(\omega b / U_{e}\right)^{1 / 2} \sin \theta .
$$

The reduced frequency $S=\omega r_{n} / U_{e}$ has been assumed $O(1)$, so that $\omega b / U_{e} \gg 1$. For $k \gg 1$, the acoustic wavelength is short compared to the airfoil chord and we obtain the quite different behaviour (HK1)

$$
\kappa_{s}=\cos \theta, \quad \kappa_{a}=2 \mathrm{e}^{-\mathrm{i} \pi / 4}\left(\pi M_{e}\right)^{-1 / 2} \sin \frac{1}{2} \theta,
$$

where $M_{e}=U_{e} / c$ is the Mach number of the mean flow, which has been assumed small. Thus, for both $k \ll 1$ and $k \gg 1, \kappa_{a}$ is much larger than $\kappa_{s}$ except when the acoustic wave incidence angle $\theta$ is small.

By writing $\psi=\kappa_{s} \psi_{s}+\kappa_{a} \psi_{a}$, and substituting into (3.9), the receptivity coefficient is given by

$$
C_{1}=\kappa_{s}(\theta) C_{s}(S, \mu)+\kappa_{a}(\theta) C_{a}(S, \mu)
$$

where $C_{s}$ and $C_{a}$ are extracted from the solutions of

$$
\left.\begin{array}{l}
\mathscr{F}\left(\psi_{s}\right)=S^{1 / 2}\left(\mathrm{i} S h^{2}(w-\mu)+\frac{\mu-2 w+\mu(w-\mu)^{2}}{h^{2}}\right), \\
\mathscr{F}\left(\psi_{a}\right)=\mathrm{i} S h^{2}-\frac{1+\mu^{2}-w^{2}}{h^{2}},
\end{array}\right\}
$$

respectively, with $\mathscr{F}$ defined in (3.9). Here $C_{s}$ is a measure of the receptivity due to that component of the free-stream disturbance which leads to symmetric flow in the vicinity of the leading edge, and $C_{a}$ is a measure of the receptivity arising from that component of the free-stream disturbance which leads to antisymmetric flow about the leading edge.

\section{Numerical results}

In $\S 3$, generalizations of the Lam-Rott asymptotic eigenfunctions were obtained, taking account of the pressure gradient due to leading-edge thickness and mean aerodynamic loading. The asymptotic analysis determines the form of these eigenfunctions, but not their coefficients $C_{i}$. The coefficients are determined by the characteristics of the free-stream disturbance in the LUBLE region, $\xi=O(1)$. The first of these eigenfunctions is the precursor of the TS wave. Thus, the free-stream disturbances influence the amplitude of the TS wave only through the coefficient $C_{1}$. It appears that the receptivity coefficient $C_{1}$ for a particular free-stream disturbance can be determined only by numerically solving the LUBLE over the full range of $\xi$ and examining the behaviour for large $\xi$.

Numerical solutions are required for both the mean flow, governed by the nonlinear partial differential equation (3.6), and the linearized disturbance, governed by the LUBLE (3.9). For convenience, the numerical solutions are computed in terms of the variable $w$ rather than $\xi$ (see (3.3)). Since the governing equations are parabolic, the solutions were computed by marching away from the mean flow stagnation point $(w=0)$ using a Keller Box scheme (Keller \& Cebeci 1970). The initial conditions at $w=0$ correspond to the steady Hiemenz flow and its quasi-steady linear perturbation.

\subsection{Mean flow}

The development of the mean flow in the leading-edge region is controlled by the mean pressure-gradient parameter $\beta$, given by (3.7). In figure $2, \beta$ is plotted as a function of 


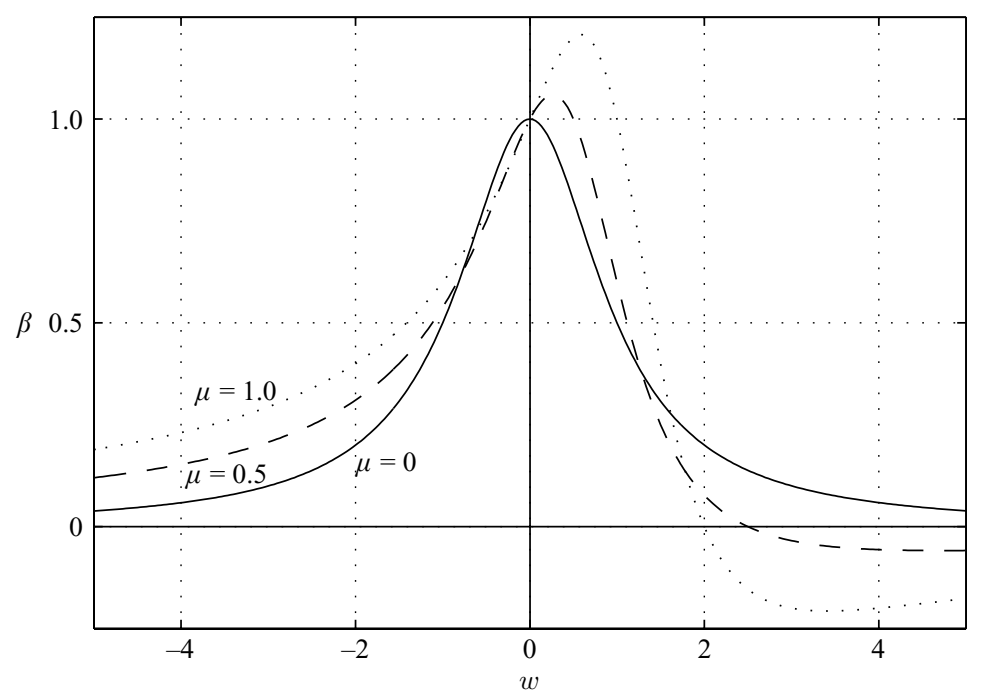

FIGURE 2. Streamwise variation of the mean pressure gradient parameter, $\beta(w)$, for $\mu=0,0.5,1.0$.

$w$ for three values of the aerodynamic loading parameter, $\mu$. For $\mu=0$, the pressure gradient is everywhere favourable, falling off monotonically away from its stagnation point value $(\beta=1)$ and approaching zero as $w^{-2}$ far downstream. The behaviour for negative $w$ (the lower surface) is identical to that for positive $w$, for $\mu=0$. When aerodynamic loading is introduced, the magnitude of the favourable pressure gradient along the upper surface is increased in the region from the stagnation point $(w=0)$ to the nose $(w=\mu)$. The pressure-gradient parameter then falls rapidly to zero at $w=\mu+\mu^{-1}$, and the pressure gradient is adverse for all locations farther downstream. Compared to the favourable pressure gradient for $\mu=0$, the adverse pressure gradient on the upper surface for $\mu>0$ decays to zero much more slowly with downstream distance, becoming proportional to $w^{-1}$ far downstream. In contrast to the complicated behaviour of the pressure gradient on the upper surface in the presence of aerodynamic loading, the pressure gradient on the lower surface $(w<0)$ remains everywhere favourable. However, when $\mu$ is non-zero the pressure gradient on the lower surface decays only as $|w|^{-1}$ for large distances, and the magnitude of the pressure gradient increases with $\mu$, except for a small region near the leading edge $(-1<w<0)$.

As the aerodynamic loading parameter is increased, the position of the minimum wall shear stress on the upper surface moves towards the nose (figure $3 a$ ), and the magnitude of the minimum wall shear decreases (figure $3 b$ ). For steady twodimensional flows, zero wall shear corresponds to boundary-layer separation. From Ruban (1982) and Stewartson, Smith \& Kaups (1982), the minimum wall shear close to the critical angle of attack is given by

$$
\phi_{\eta \eta}\left(w_{m}, 0\right) \propto\left(\mu_{c}-\mu\right)^{1 / 2} .
$$

Fitting this behaviour to the curve in figure $3(b)$ suggests that separation occurs at $\mu_{c} \approx 1.15$. More accurate determination of separation criteria would require a modified numerical scheme, since the current method breaks down near the onset of flow reversal. 

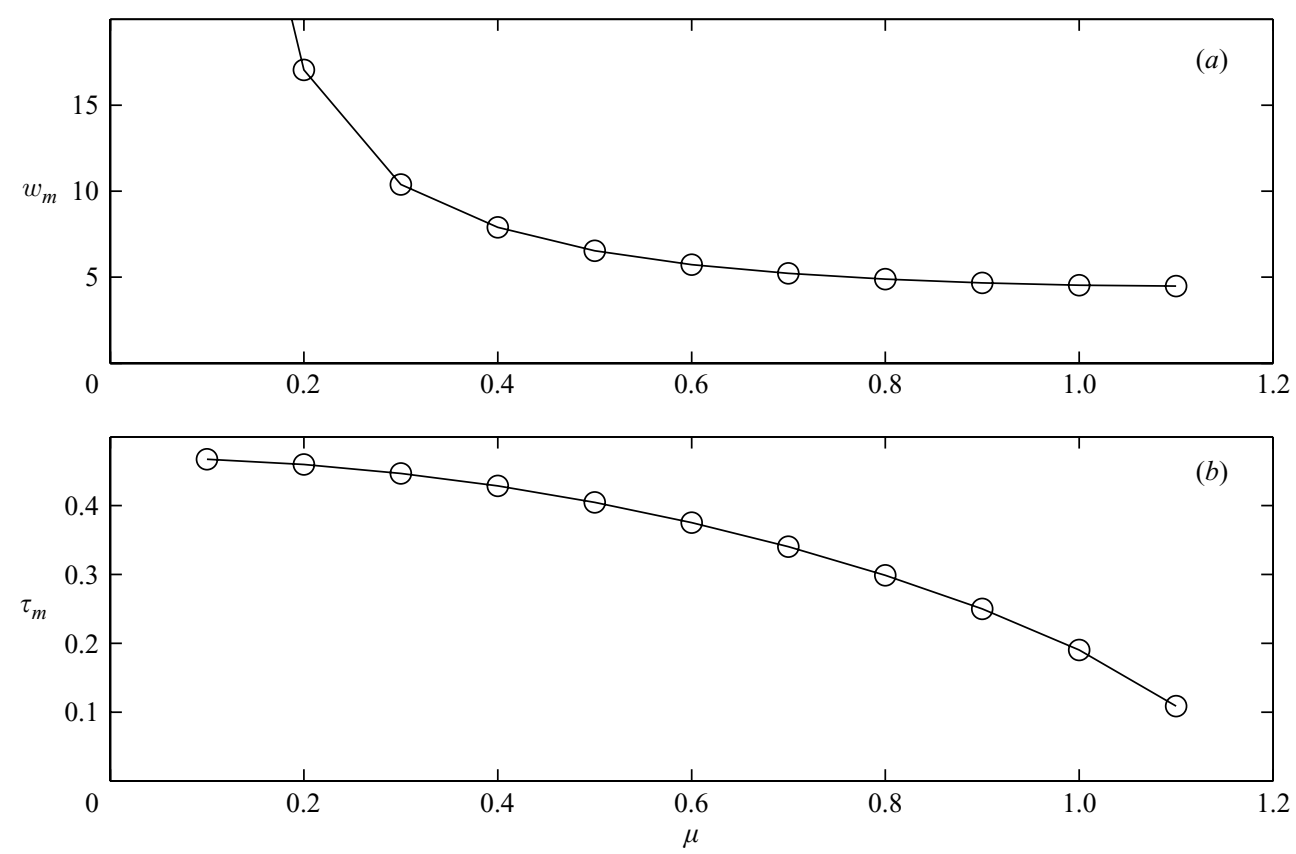

FIGURE 3. Effect of aerodynamic loading parameter on the wall shear stress: $(a)$ position of minimum wall shear, $w_{m} ;(b)$ magnitude of minimum wall shear, $\phi_{\eta \eta}\left(w_{m}, 0\right)$.

The coefficient $B_{1}(\mu)$, which appears in the functions $p_{3}(\eta)$ and $p_{5}(\eta)$ arising in the large- $w$ asymptotic form of the mean flow (3.12), is undetermined by the asymptotic analysis. The value of $B_{1}$ is related to the 'virtual origin' of the boundary layer. This can be seen from (A 6), by noting that the function $\eta F^{\prime}-F$ which $B_{1}$ multiplies in $p_{3}$ corresponds to the first eigensolution of Libby \& Fox (1963). Their first eigensolution is the streamwise derivative of the streamfunction for the Blasius boundary layer. The value $B_{1}(0)$ relates to a shift of virtual origin due to the favourable pressure gradient on the nose of the parabola in the absence of aerodynamic loading; the dependence on $\mu$ relates to an additional shift of the virtual origin in the presence of aerodynamic loading.

To determine $B_{1}(\mu)$, the wall shear $U_{0}^{\prime}(w)=\phi_{\eta \eta}(w, 0)$ predicted by the asymptotic expansion (A 7) is compared with the wall shear obtained by numerical solution of (3.6), for each value of $\mu$ considered. In fact it proves necessary to modify (3.6) and solve for $\phi(w, \eta)-F(\eta)$ in order to obtain the required accuracy when extrapolating for $B_{1}$ in the large- $w$ limit. In figure 4 , it is seen that $B_{1}(0) \approx 2.08$ and that $B_{1}(\mu)$ increases approximately linearly with $\mu$ over the range $-0.4<\mu<0.7$. The maximum value of $B_{1}(\mu)$ occurs at $\mu \approx 0.9 ; B_{1}(\mu)$ decreases slightly for larger values of $\mu$.

\subsection{Unsteady flow}

The value of the receptivity coefficient $C_{1}(S, \mu)$, for a particular free-stream disturbance, is obtained by comparing numerical solutions of the LUBLE to the asymptotic forms (3.17) and (3.18).

For typical values of the camber distribution and angle of attack, the mean aerodynamic loading parameter $\mu$ is positive. We examine the influence of mean aerodynamic loading on the receptivity for both surfaces of the airfoil. However, rather than considering the cases $w>0$ (upper surface) and $w<0$ (lower surface) 


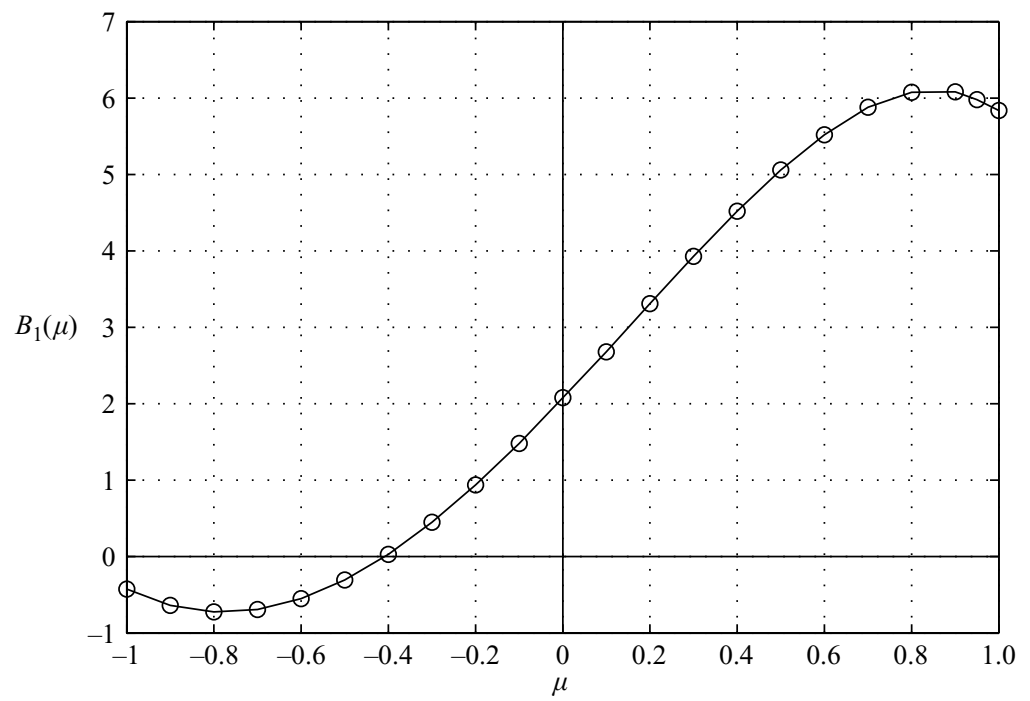

FIGURE 4. Computed values of $B_{1}(\mu)$, the coefficient appearing in the expansion of the base flow far downstream (A 7).

separately, the results can be presented more concisely by considering $w$ to be positive and taking $\mu>0$ to give upper surface results and $\mu<0$ for lower surface results.

The method used to obtain numerical values for $C_{s}$ and $C_{a}$ is described in HK1. For real $w$, the real part of $T_{i}^{(0)}(w)$ is negative. Thus, the asymptotic eigenfunctions (3.15) decay exponentially with downstream distance, while the particular solution $\psi_{p}$ remains $O(1)$. In addition, the eigenfunctions are inverse ordered, so that the function $\psi_{1}$ whose coefficient is desired decays exponentially faster than all the other eigenfunctions. To circumvent these difficulties, the streamwise integration is extended into the complex plane in order to make the first asymptotic eigenfunction dominant. Choosing $-5 \pi / 12<\arg (w)<-\pi / 12$, the first eigenfunction becomes exponentially large compared to the other components of the unsteady boundary layer solution for $\left|S^{1 / 2} w\right| \gg 1$. However, the pressure gradient parameter $\beta$, defined by (3.7), has first-order poles at $w=\mu \pm \mathrm{i}$. Thus for larger values of $\mu, \arg (w)$ must be chosen with care in order to avoid an integration path which passes close to the singularity at $w=\mu-\mathrm{i}$. For all values of $\mu$ considered, integration was performed for $\arg (w)=-0.1 \pi$ and $\arg (w)=-0.15 \pi$; for smaller values of $\mu$, results for $\arg (w)=-0.2 \pi$ and $\arg (w)=-0.25 \pi$ were also obtained. For these choices of $\arg (w)$, the solution $\psi$ grows exponentially with $|w|$.

To avoid difficulties associated with the rapid exponential growth of $\psi$ as a function of $|w|$, in our numerical scheme we solve for $\widehat{\psi}=\psi \exp \left(-T_{1}^{(0)}\right)$ rather than $\psi$. This allows the numerical solution to be continued much farther downstream while retaining accuracy (Heinrich \& Kerschen 1989). Using the wall shear (3.17) as the basis for comparison, we define

$$
\mathscr{C}(\mu, S, w)=\frac{\widehat{\psi}_{\mathrm{Num}}^{\prime \prime}(\eta=0)}{\widehat{\psi}_{0}^{\prime \prime}},
$$

where $\widehat{\psi}_{0}^{\prime \prime}=0.4356(1+i)\left(S w^{2} / 2\right)^{\tau_{1}+1}$. Since the argument of $w$ was chosen so that the first asymptotic eigenfunction dominates other components of the solution when 
$w \gg 1, \widehat{\psi} \sim C_{1} \psi_{1} \exp \left(-T_{1}^{(0)}\right)$ and hence

$$
\mathscr{C} \sim C_{1}\left[1+c_{1} \frac{1}{w^{0.774}}+c_{2} \frac{\ln ^{2} w}{w}+c_{3} \frac{\ln w}{w}+c_{4} \frac{1}{w}+\ldots\right] \text { for } w \gg 1 .
$$

The value of $C_{1}(\mu, S)$ is then obtained by extrapolation, though this is complicated by the presence of four closely spaced terms.

The development of the unsteady disturbance along a ray in the complex $w$ plane contains two phases, both of which must be calculated accurately in order to determine values of the receptivity coefficient. First, over a relatively short distance, the asymptotic eigenfunction $\psi_{1}$ grows exponentially relative to the other components of the solution and becomes the dominant component. In our computations, this stage is typically completed when $S^{1 / 2} w \approx 3$. (This is seen by plotting $\mathscr{C}(\mu, S, w)$, defined in (5.1), as a function of $|w|$.) Second, over a much longer distance the asymptotic eigenfunction evolves algebraically toward its large- $w$ form (3.15). The approach of $\psi_{1}$ to its large- $w$ form is much slower for larger values of $\mu$, due to the long downstream distance required for the mean boundary layer to recover from the region of low wall shear induced by the strong adverse pressure gradient. Computations were typically carried out to distances $S^{1 / 2} w \approx 15-20$, except for larger values of $\mu$ when it was necessary to extend the computations farther downstream.

For the larger values of $\mu$ and $S$ considered, a small error in the computed value of $B_{1}$ leads to a large error in $\mathscr{C}(\mu, S, w)$ for $w \gg 1$, and hence limits the accuracy of the extrapolated value of $C_{1}$. In the analysis of HK1 for a symmetric airfoil in the absence of aerodynamic loading $(\mu=0)$, arg $w=-0.25 \pi$ was used throughout. With this particular choice, any error in the extrapolated value of $\left|C_{1}\right|$ due to inaccuracy in the numerically determined value of $B_{1}$ is eliminated. Clearly this advantage is lost in the present study. However, $C_{1}$ should be independent of the value of $\arg w$ used in the numerical integration. By using two different values of $\arg w$ in the present analysis, any significant error due to inaccurate determination of $B_{1}$ should be readily identifiable. Unfortunately, for the larger values of $\mu$ and $S$ considered, there is still uncertainty in the numerical value of $C_{1}$ obtained by extrapolation. For $\mu=0.9$, the error may be as large as $25 \%$.

\subsection{Receptivity coefficients}

In figure 5, the moduli of the receptivity coefficients are plotted as a function of the mean loading parameter $\mu$, for values which correspond to the upper surface $(\mu>0)$. The coefficients $\left|C_{s}\right|$ and $\left|C_{a}\right|$ for free-stream disturbances symmetric and antisymmetric about the nose of the body, respectively, are plotted in figures $5(a)$ and $5(b)$. Results are presented for two Strouhal numbers, $S=0.1$ and $S=0.3$. The overall patterns of the variation with the aerodynamic loading parameter $\mu$ are qualitatively similar for $\left|C_{s}\right|$ and $\left|C_{a}\right|$. However, the receptivity coefficient for antisymmetric forcing is larger than that for symmetric forcing, by approximately a factor of two (note the different scales in figures $5 a$ and $5 b$ ). As the aerodynamic loading parameter is increased from zero, the receptivity level on the upper surface $(\mu>0)$ decreases; $\left|C_{s}\right|$ reaches a minimum at $\mu \approx 0.7$, while $\left|C_{a}\right|$ reaches a minimum at $\mu \approx 0.4$. Beyond these minima the receptivity coefficients $\left|C_{s}\right|$ and $\left|C_{a}\right|$ increase with $\mu$, reaching local maxima in the vicinity of $\mu \approx 0.9$. The quantitative accuracy of the numerical results for values of $\mu$ beyond 0.9 is uncertain, for the reasons discussed earlier.

The variations in both $\left|C_{s}\right|$ and $\left|C_{a}\right|$ as a function of $\mu$ are much larger for $S=0.3$ than for $S=0.1$. For $S=0.3$, the receptivity levels on the upper surface drop 

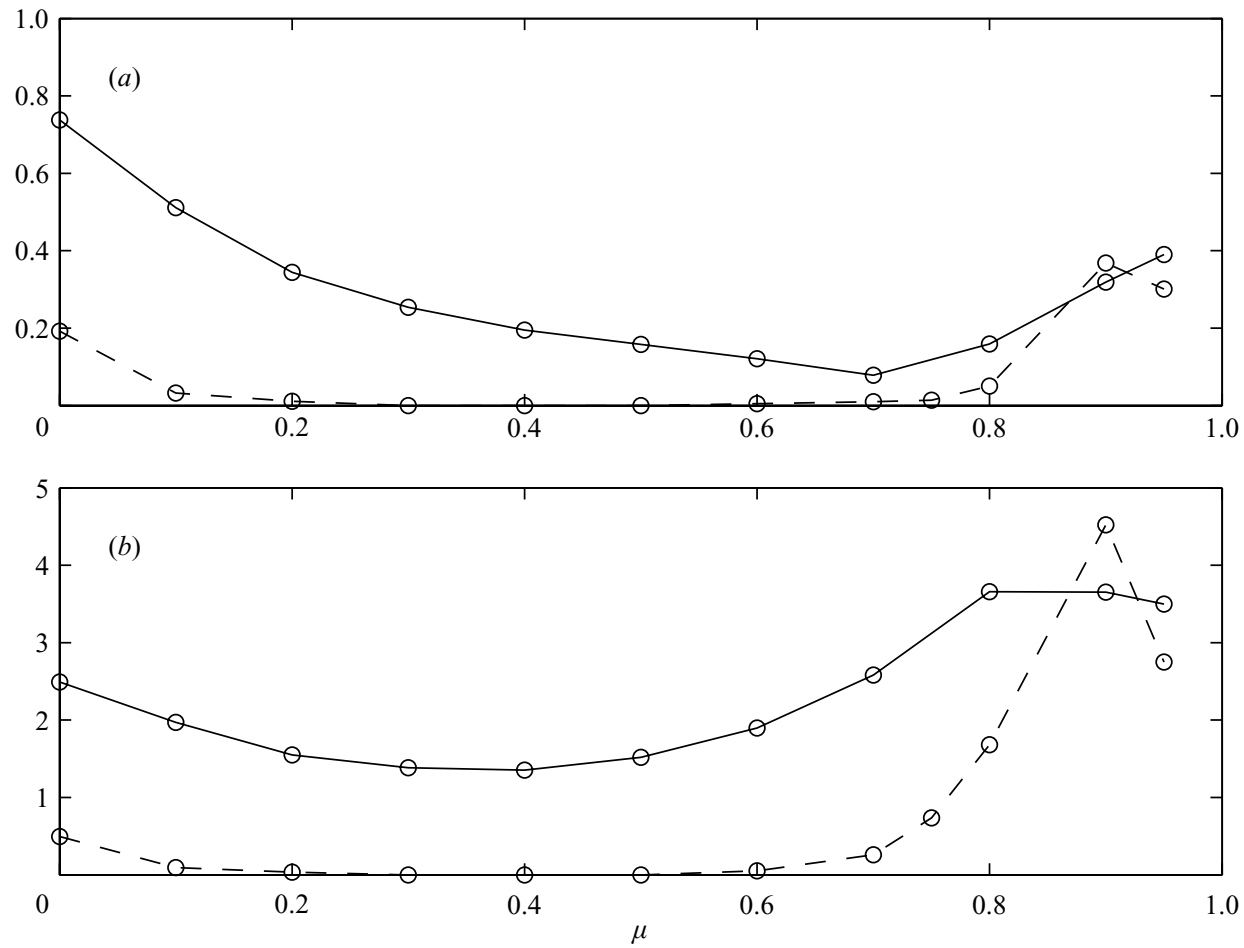

FIGURE 5. Variation of the magnitude of the receptivity coefficient on the upper surface of the body as a function of the aerodynamic loading parameter $\mu$, for two Strouhal numbers, $S=0.1$ (solid line) and 0.3 (dashed line). (a) Receptivity to the free-stream disturbance component symmetric about the nose, $\left|C_{s}\right| ;(b)$ receptivity to the free-stream disturbance component antisymmetric about the nose, $\left|C_{a}\right|$.

significantly for modest values of aerodynamic loading. Both $\left|C_{s}\right|$ and $\left|C_{a}\right|$ are less than 0.02 in the region $0.2<\mu<0.6$. (It is not possible to provide precise values in this region, because the errors that arise in extrapolation are absolute rather than relative.) The subsequent rise in receptivity level near $\mu \approx 0.9$ is particularly striking for the case of antisymmetric forcing with $S=0.3$, where the local value of $\left|C_{a}\right|$ exceeds 4.

The presence of aerodynamic loading adds significant complexity to the development of the unsteady flow, especially on the upper surface where the boundary layer first experiences a rapid acceleration, followed by a rapid deceleration and then a slow recovery. Thus it is difficult to provide a simple explanation for the dependence of the receptivity coefficient on the aerodynamic loading parameter. For the symmetricmean-flow case $(\mu=0)$ considered in HK1, the mean pressure gradient is everywhere favourable, monotonically decreasing from its maximum value at the stagnation point to zero far downstream as seen in figure 2. An increase of the leading-edge nose radius (or Strouhal number) was found to decrease the receptivity coefficient. As a speculative explanation of this behaviour, it was suggested that a favourable pressure gradient in the region where the receptivity occurs tends to reduce the receptivity level. At larger values of $S$, the region $\xi=O(1)$ where the receptivity occurs is concentrated closer to the airfoil nose, in the region of higher favourable pressure gradient. Hence, the decrease in receptivity for increasing $S$ found in HK1 is consistent with this 
explanation. However, the rise in receptivity level in the vicinity of $\mu \approx 0.9$ is not explained by this simple explanation. Referring back to the streamwise variation in mean wall shear in figure 3 , we see that this value of mean loading corresponds to the minimum wall shear becoming small. We now consider the possibility that the region of low wall shear contributes to the total receptivity on the upper surface.

As discussed in $\S 1$, receptivity comes about through the interaction of free-stream disturbances with regions of the boundary layer that contain short-scale streamwise gradients. In the leading-edge mechanism, the receptivity is concentrated in a region of length $O\left(U_{e} / \omega\right)$ extending downstream from the mean-flow stagnation point. However, for values of $\mu$ approaching the critical value for the onset of separation, $\mu_{c} \approx 1.15$, additional short-scale streamwise variations develop in the mean flow in the vicinity of the location of minimum wall shear, $w=w_{m}$. From figure 3 , it is seen that $w_{m} \approx 5$ for values of $\mu \geqslant 0.8$. The corresponding distance from the mean-flow stagnation point is $l_{m} \approx 9 r_{n}$, so that $\omega l_{m} / U_{e} \approx 9 S$. Thus, for low values of $S$, the minimum wall shear point lies within the leading-edge receptivity region, and the receptivity which occurs in the vicinity of $w_{m}$ can be calculated by the present method, while at higher values of $S$ the minimum wall shear point lies downstream of the leading-edge receptivity region.

Applying the marginal separation receptivity analysis of Goldstein et al. (1987) to the present problem requires that $\mu_{c}-\mu=O\left(\epsilon^{14 / 5}\right)$ and $S=O\left(\epsilon^{-1}\right)$. The scaling of $\mu$ leads to an interactive viscous-inviscid structure for the mean flow in the vicinity of $w_{m}$, but with a local streamwise scale of $O\left(\epsilon^{2}\right)$ as compared to the $O\left(\epsilon^{3}\right)$ scale of standard triple-deck theory. This leads to additional complexity for the marginal separation receptivity analysis, compared to the localized receptivity analysis of Goldstein (1985) in which the variations in surface geometry and the TS wavelength both have the standard triple-deck scale. Specifically, in Goldstein's marginal separation analysis, the instability waves in the marginal separation region are interactive but their wavelength is shorter than the local scale for the mean flow. This leads to an exponentially small initial amplitude for the instability wave. However, the wave undergoes exponential amplification in the marginal separation region and somewhat downstream, so that the marginal separation receptivity mechanism could be important in some applications.

Our analysis assumes a high Reynolds number $(\epsilon \ll 1)$ with $\mu_{c}-\mu=O(1)$, so that the mean flow remains non-interactive in the region of low wall shear. Thus, we cannot make direct comparisons with the analysis of Goldstein et al. (1987). However, as discussed above, at sufficiently low Strouhal numbers the region of low wall shear is contained within the leading-edge receptivity region, which is treated by our theory. In this case, we can investigate the relative importance of the receptivity in the region of low wall shear, compared to the receptivity near the mean-flow stagnation point. The streamwise distribution of the receptivity process, for a range of values of $\mu$ and $S$, is examined in the following subsection. However, for completeness, results for the receptivity on the lower surface of the airfoil are first given.

The receptivity on the lower surface of the airfoil is illustrated in figure 6. On the lower surface $(\mu<0)$, the receptivity coefficients $\left|C_{s}\right|$ and $\left|C_{a}\right|$ increase as aerodynamic loading is introduced, to global maxima in the vicinity of $\mu \approx-0.4$. The receptivity coefficients then decrease toward zero as $\mu$ approaches -1 . For negative $\mu$, the value of the receptivity coefficient can be extracted from the computational results with a good degree of accuracy, since the pressure gradient is everywhere favourable and decays monotonically to zero, with no region of low wall shear. 

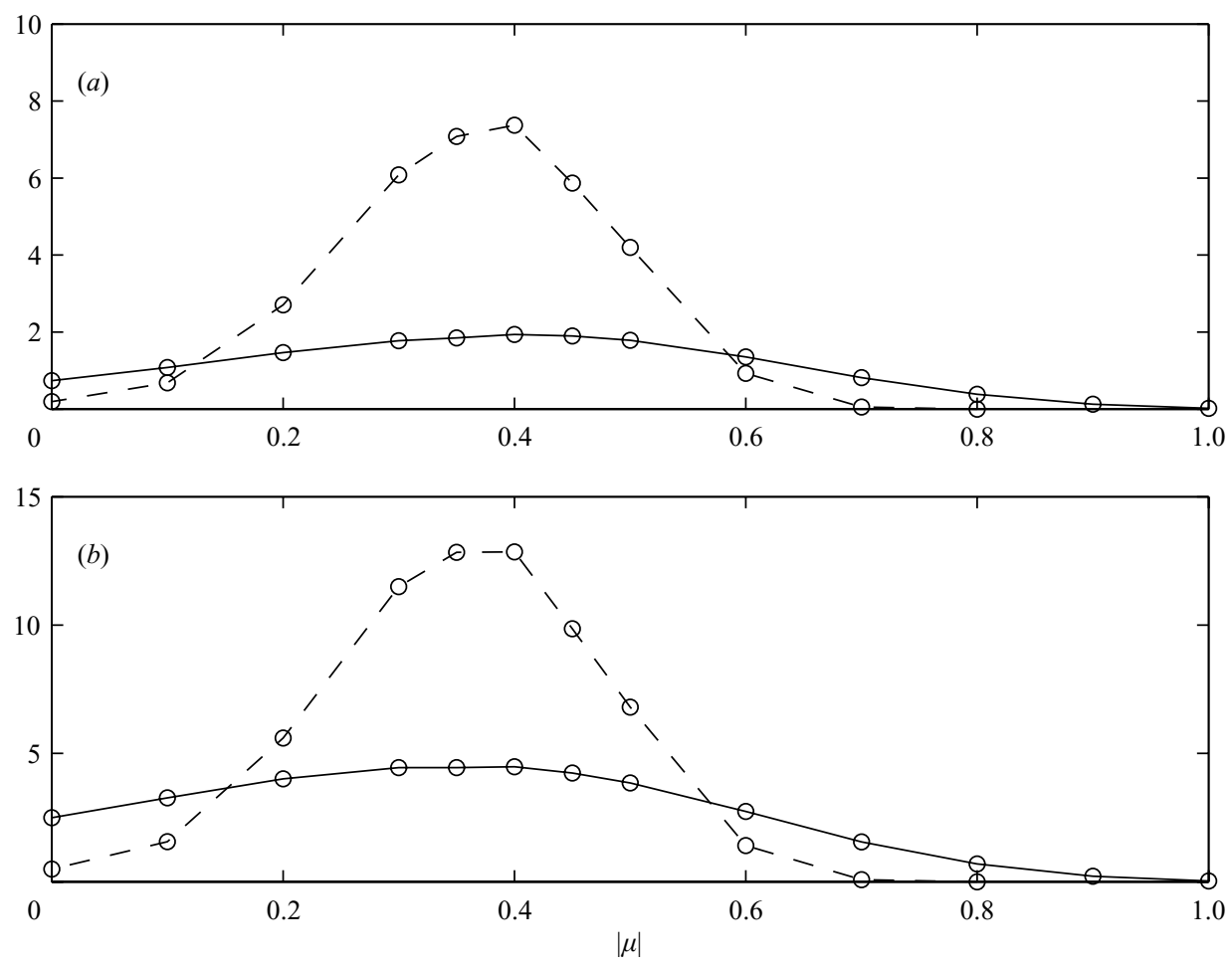

FIGURE 6. Variation of the magnitude of the receptivity coefficient on the lower surface of the body for $S=0.1$ (solid line) and 0.3 (dashed line) due to the free-stream disturbance component $(a)$ symmetric about the nose; $(b)$ antisymmetric about the nose.

The large values found for $\left|C_{s}\right|$ and $\left|C_{a}\right|$ in the vicinity $\mu \approx-0.4$ imply that the instability wave on the lower surface of the airfoil has a larger 'initial' amplitude for this value of aerodynamic loading. The transition point, however, is determined by both the receptivity coefficient and the stability characteristics. On the lower surface, the high receptivity coefficient is counteracted by the increased stability due to the favourable pressure gradient (see figure 2). Note also that the instability wave amplitude depends linearly on the receptivity coefficient, while it depends exponentially on the instability wave growth rate. Thus, despite the larger receptivity coefficient on the lower surface, the transition point on the lower surface in the presence of aerodynamic loading is typically farther downstream than that on the upper surface.

The objective of a receptivity analysis is to provide the 'initial amplitude' which is required in order to compute the subsequent downstream development of the instability wave, and possibly the eventual transition of the boundary layer. For $S^{1 / 2} w \gg 1$, the first asymptotic eigenfunction $\psi_{1}$ of the LUBLE evolves to the initial form of the TS wave of the Orr-Sommerfeld equation. Thus, the initial amplitude of the TS wave is determined by $C_{1} \exp \left(T_{1}^{(0)}(w)\right)$, where $T_{1}^{(0)}(w)$ is given by the asymptotic analysis and numerical values of $C_{1}$ are given in figures 5 and 6 . This information is sufficient to provide upstream boundary conditions for stability codes in order to investigate the position of transition. However in order to understand the process of receptivity further, it is still instructive to consider the relative importance of the streamwise regions which contribute to the total receptivity. 


\subsection{Streamwise distribution of the receptivity process}

The development of the unsteady field involves a number of processes. First, there is a transfer of energy from the free-stream disturbance to the viscous motion in the boundary layer, through the action of the unsteady slip velocity and pressure gradient imposed by the free-stream disturbance. Second, there is a 'filtering' process within the boundary layer, which determines the coefficients $C_{i}$ of the generalized Lam-Rott asymptotic eigenfunctions, $\psi_{i}$. Third, there is a phase in the development of the unsteady flow in which a strong disparity develops in the relative magnitudes of the asymptotic eigenfunctions, due to their differing exponential decay (or growth) rates. Fourth, there is an algebraic evolution of $\psi_{i}$ to its far downstream form.

The question then arises of the extent to which these various processes or phases are distinct. We believe that there is no sensible way to separate the first and second processes - in our view the 'leading-edge receptivity process' is a combination of the transfer of energy from the free-stream disturbance and its filtering within the boundary layer to determine the coefficient $C_{1}$. The asymptotic theory shows that this process takes place in the LUBLE region where $\omega l / U_{e}=O(1)$, where $l$ is the dimensional distance along the airfoil measured from the stagnation point.

In contrast to the first two processes, which cannot be separated, the third and fourth processes are distinct phases in the streamwise development of the unsteady flow. The third process, in which $\psi_{1}$ becomes the dominant eigenfunction (when the computation is carried out along a ray in the complex $w$-plane), occurs over a relatively modest downstream distance extending out to, say $S^{1 / 2} w \approx 3\left(\omega l / U_{e} \approx 4\right)$, as could be predicted by noting that the relative magnitudes of the first two eigenfunctions, $\psi_{1}$ and $\psi_{2}$, are given by $\exp (T(w))$ where $\rho$ is replaced by $\rho_{1}$ and $\rho_{2}$, respectively (see Appendix B).

The fourth process, of algebraic evolution to the final downstream form for large $S^{1 / 2} w$, extends over a much larger downstream distance. For the case $\mu=0$ where the favourable pressure gradient decays as $w^{-2}$, the fourth process extends out to distances of the order of $\omega l / U_{e} \approx 20$. For non-zero values of aerodynamic loading, the pressure gradient decays as $w^{-1}$ and the fourth process extends even farther downstream. For larger values of $\mu$, the adverse pressure gradient on the upper surface leads to an extended region of low wall shear stress and the recovery of the mean boundary layer to Blasius form is extremely slow. The algebraic evolution of the Lam-Rott eigenfunction to its final downstream form cannot precede the recovery of the mean boundary layer, so that in this case the fourth process in the evolution of the unsteady field extends very far downstream, say to $\omega l / U_{e} \approx 100$ for $\mu \approx 0.9$. The large downstream distance required for the unsteady motion to evolve algebraically to the asymptotic eigenfunction $\psi_{1}$, combined with rapid exponential growth (or decay) throughout this region, makes it quite difficult to obtain highly accurate numerical results in this region of parameter space.

The third and fourth processes both take place in the far downstream asymptotic limit of the LUBLE region, $1 \ll \omega l / U_{e} \ll \epsilon^{-2}$. In this downstream region, the asymptotic eigenfunctions are decoupled from the local values of the free-stream disturbance, so that the 'receptivity process' (as defined earlier) has been completed.

Focusing on the region of receptivity $\left(\omega l / U_{e}=O(1)\right)$, it is interesting to examine quantitatively the extent of the region in which a significant transfer of energy from the free-stream disturbance to the asymptotic eigenfunction $\psi_{1}$ takes place. In addressing this question, we take advantage of the fact that the equation governing the unsteady component of the flow is linear. Hence, we can consider the receptivity coefficient $C_{1}$ (or $C_{s}$ and $C_{a}$ ) as arising from a superposition of the contributions 
from the free-stream disturbance at various values of $w$. By removing the freestream disturbance input at downstream locations and examining the influence on the receptivity coefficient, we can determine the relative importance of the receptivity at these downstream locations. Note that here we are focusing on the relative receptivity for different free-stream disturbance functions, and this can be calculated very accurately even when $\mu$ is large. Essentially, the calculations need only be carried out to the stage where the first asymptotic eigenfunction becomes (exponentially) dominant, since the subsequent algebraic evolution of $\psi_{1}$ to its far downstream form is independent of the free-stream disturbance in this downstream region.

To investigate the relative contribution of different downstream regions to the total receptivity, we consider a modified slip velocity

$$
\widehat{u}_{s}(w)=u_{s}(w) \Phi\left(w, w_{0}\right)
$$

where

$$
\Phi\left(w, w_{0}\right)=\frac{1-\tanh (\zeta)}{1-\tanh \left(\zeta_{0}\right)}, \quad \zeta=\frac{w-w_{0}}{\delta_{w}}, \quad \zeta_{0}=-\frac{w_{0}}{\delta_{w}},
$$

is a smooth transition function that removes the contribution from the free-stream disturbance at downstream locations. The modified slip velocity is the same as the physical slip velocity for small values of $w$, then decreases smoothly and monotonically to zero in the vicinity of $w=w_{0}$. The transition occurs over a $w$-scale of $\delta_{w}$. The corresponding unsteady pressure gradient is then given by

$$
\frac{\mathrm{d} \widehat{p}}{\mathrm{~d} w}=\Phi \frac{\mathrm{d} p}{\mathrm{~d} w}-\frac{w u_{s}}{h} \frac{\partial \Phi}{\partial w}
$$

and we extract the modified receptivity coefficients $\widehat{C}_{s}\left(\mu, S, w_{0}, \delta_{w}\right)$ and $\widehat{C}_{a}\left(\mu, S, w_{0}, \delta_{w}\right)$ from the solutions of

$$
\left.\begin{array}{l}
\mathscr{F}\left(\widehat{\psi}_{s}\right)=S^{1 / 2}\left\{\Phi\left(\mathrm{i} S h^{2}(w-\mu)+\frac{\mu-2 w+\mu(w-\mu)^{2}}{h^{2}}\right)-\Phi_{w} w(w-\mu)\right\}, \\
\mathscr{F}\left(\widehat{\psi}_{a}\right)=\Phi\left(\mathrm{i} S h^{2}-\frac{1+\mu^{2}-w^{2}}{h^{2}}\right)-\Phi_{w} w,
\end{array}\right\}
$$

respectively. By varying $w_{0}$, the relative contribution of different streamwise regions to the total receptivity can be determined. However, the introduction of a new artificial streamwise scale (i.e. $\delta_{w}$ ) clearly provides another possible source of receptivity. Thus care must be taken in choosing $\delta_{w}$ : small enough that the different regions of receptivity can be differentiated, but not so small that the receptivity associated with the rapid change in unsteady slip velocity dominates other contributions to the receptivity. Moreover, $\Phi$ is singular when $w=w_{0}-\frac{1}{2} \pi \delta_{w}$ i. Hence our choice of $\delta_{w}$ is restricted by the condition $\delta_{w}>2 w_{0} \tan [\arg (w)] / \pi$, which ensures that the integration contour does not pass through any such singularity.

To examine the contributions to the receptivity on the upper surface when the body is close to the critical angle of attack, we consider the ratio

$$
\Gamma\left(\mu, S, w_{0}, \delta_{w}\right)=\frac{\widehat{C}\left(\mu, S, w_{0}, \delta_{w}\right)}{C(\mu, S)}
$$

for $\delta_{w}=3.0$ and for various values of $\mu$. By definition, $\Gamma \rightarrow 1$ as $w_{0} \rightarrow \infty$. Considering $\Gamma$ as a function of $w_{0}$, the point at which $\Gamma$ approaches 1 gives the downstream point at which all coupling between the free-stream disturbance and the instability wave 

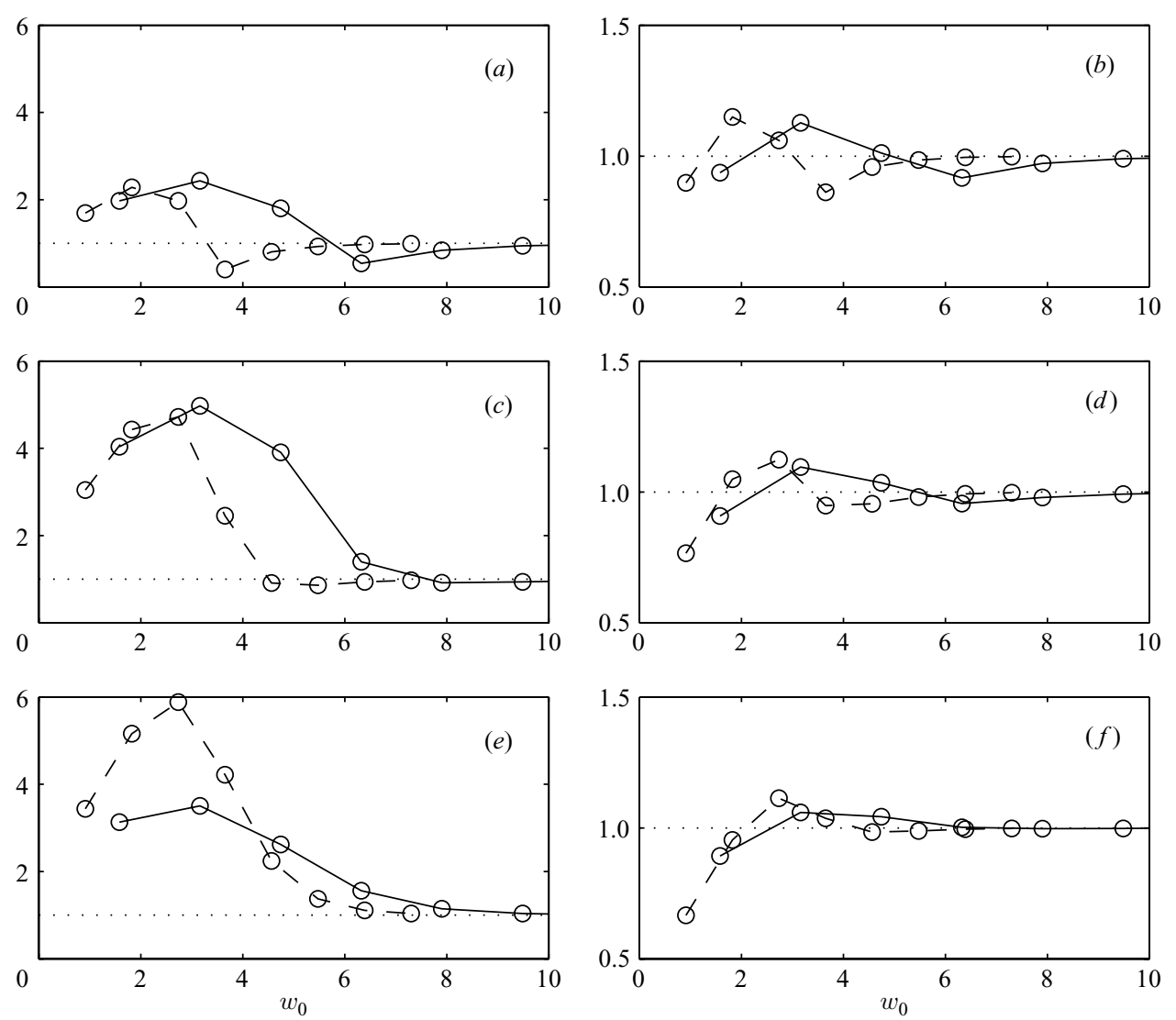

FIGURE 7. Plot of $|\Gamma|$ illustrating the relative receptivity as a function of the streamwise location of the filter based on body geometry, $w_{0}$, for $\mu=0,0.5$ and 0.9 for the component of the disturbance symmetric about the nose $(a, c, e)$ and for the component of the disturbance antisymmetric about the nose $(b, d, f)$. Solid lines mark the results for $S=0.1$, and dashed lines mark the results for $S=0.3$.

has ceased. In the following discussion we will refer to this as the point at which receptivity is complete.

In figure 7, results for $|\Gamma|$ are plotted as a function of the filter switch-off point $w_{0}$, for two Strouhal numbers $S=0.1$ and 0.3 . The value of $|\Gamma|$ is seen to approach 1 as $w_{0}$ increases, indicating the completion of the receptivity process. The receptivity region would be expected to be concentrated closer to the mean flow stagnation point at the higher frequency $S=0.3$, and this behaviour is indeed seen in the plot of $\left|\Gamma_{s}\right|$. Upon closer inspection, a similar trend can be seen in the plot of $\left|\Gamma_{a}\right|$.

A possible mechanism explaining the rise in receptivity coefficient for $\mu \approx 0.9$ is the appearance of a region of low wall shear as discussed earlier in $\S 5.3$. However, for $\mu=0.9$, the point of minimum wall shear occurs at $w=4.67$. For $S=0.3$, the results in figure 7 show that the receptivity process has been completed by the time the region of low wall shear is reached. Thus, these results indicate that the dramatic increases in $\left|C_{s}\right|$ and $\left|C_{a}\right|$ near $\mu \approx 0.9$ in figure 5 are not due to receptivity in the region of low wall shear. A further observation, which relates to the lack of receptivity in the region of low wall shear for $S=0.3$, is that the increase in $\left|C_{a}\right|$ near $\mu \approx 0.9$ is much larger than the increase in $\left|C_{s}\right|$, even though the amplitude of the antisymmetric 
component of the slip velocity (4.1) at $w=4.67$ is only about a quarter of the value for the symmetric component of the slip velocity.

For $S=0.1,\left|\Gamma_{s}\right|$ does not approach the value of 1 until $w_{0}$ is approximately 7 . Thus, in this case the region of low wall shear appears to make some contribution to the total receptivity. This is not unexpected, since the region of receptivity $\left(\omega l / U_{e}=O(1)\right)$ extends farther downstream by a factor of 3 for the case $S=0.1$, as compared to the case $S=0.3$. Note also that $\left|\Gamma_{a}\right|$ approaches the value of 1 at a smaller value of $w_{0}$ than that for $\left|\Gamma_{s}\right|$. This may be explained by the different behaviour of the symmetric and antisymmetric components of the slip velocity as a function of downstream distance, as noted at the end of the preceding paragraph. However, although the receptivity to the symmetric free-stream disturbance extends out through the region of low wall shear for the case $S=0.1$, there is no indication in figure 7(a) of a concentration of receptivity in the region of low wall shear. It is also interesting to note that $\left|\Gamma_{s}\right|$ is not a monotonically increasing function of $w_{0}$. This feature indicates that, at least for $\left|\Gamma_{s}\right|$, receptivity close to the leading edge is partially cancelled by receptivity at positions somewhat farther downstream. We should note that some caution is necessary in evaluating the results for $S=0.1$, since in that case the wavelength of the unsteady motion is longer and the results could have been influenced more by the finite value of the filter width $\delta_{w}$.

The results presented in figure 7 show that the receptivity region extends farther downstream at lower values of the Strouhal number. In order to examine the extent to which it is possible to scale this feature out of the results, in figure 8 we plot $\left|\Gamma_{s}\right|$ and $\left|\Gamma_{a}\right|$ as a function of $S^{1 / 2} w_{0}$, a streamwise coordinate scaled on the receptivity-region length scale $U_{e} / \omega$. Results are presented for two values of the Strouhal number, $S=0.1$ and 0.3 . The case $\mu=0$ is shown in figure $8(a, b)$ for symmetric and antisymmetric forcing respectively. Here, plotting the results in terms of a variable scaled by the receptivity length scale has produced an excellent collapse of the results for the two values of $S$. In figure $8(c, d)$ for the case $\mu=0.5$, the collapse is not quite as impressive, with the curve for $S=0.3$ extending out to a slightly larger value of $S^{1 / 2} w_{0}$ before approaching the value of 1 . This trend which appeared in figure $8(c, d)$ is magnified further in figure $8(e, f)$, where results for $\mu=0.9$ are presented. However, in all cases the leading-edge receptivity process is completed by the time a downstream distance of $S^{1 / 2} w_{0}=3$ is reached.

A number of factors may contribute to the lack of collapse in figure $8(e, f)$, where $\mu=0.9$. First, as $\mu$ is increased, the similarity behaviour for the mean boundary layer as a function of $S$ is progressively lost. Second, the strong acceleration of the flow between the stagnation point and the nose of the airfoil may introduce a significant shift in the 'virtual origin' of the boundary layer, which could affect the streamwise development of the receptivity process. Thus, it seems unlikely that a collapse of the results for different frequencies could be achieved for cases with significant aerodynamic loading.

Similar calculations were performed for $\mu=-0.4$ and -0.8 . The results for $|\Gamma|$ indicate that the receptivity on the lower surface is also concentrated in the region $S^{1 / 2} w_{0}<3$. The calculations did not produce any features which provide an explanation for the high receptivity levels in the vicinity of $\mu=-0.4$. However, as noted earlier, the numerical value of the receptivity coefficient on the lower surface is of less physical importance due to the increased stability of the boundary layer.

Returning to the question of the importance and role of the region of low wall shear at larger values of $\mu$, further insight can be gained by comparing the length scale of the mean boundary layer flow to the wavelength of the first eigensolution. 

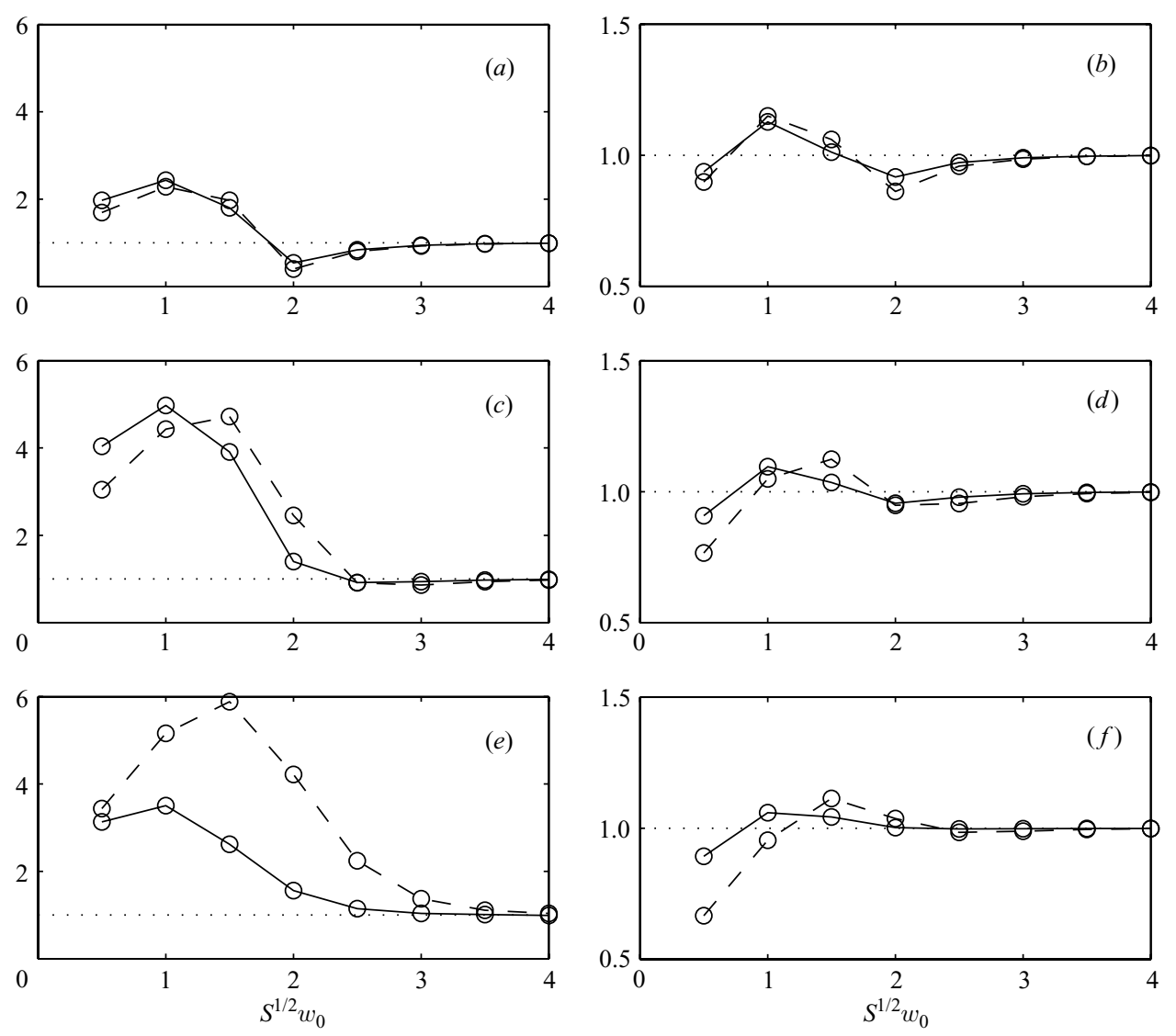

FIGURE 8. Plot of $|\Gamma|$ illustrating the relative receptivity as a function of the streamwise location of the filter, $\sqrt{S} w_{0}$, based on the receptivity length scale: $(a, c, e)$ symmetric, $(b, d, f)$ antisymmetric; $(a, b) \mu=0,(c, d) \mu=0.5,(e, f) \mu=0.9$.

We write these expressions in terms of the streamwise distance from the stagnation point, non-dimensionalized by the nose radius $r_{n}$, which in our notation is given by

$$
s=\widehat{s}(\bar{\xi})+\widehat{s}(\mu), \quad \widehat{s}(\bar{\xi})=\frac{1}{2}\left[\bar{\xi}\left(1+\bar{\xi}^{2}\right)^{1 / 2}+\log \left(\bar{\xi}+\left(1+\bar{\xi}^{2}\right)^{1 / 2}\right)\right]
$$

for a parabolic body. A reasonable choice for the streamwise length scale of the mean boundary layer is

$$
L(s)=\frac{\bar{\tau}}{\mathrm{d} \bar{\tau} / \mathrm{d} s}, \quad \bar{\tau}=\frac{w \phi_{\eta \eta}(w, 0)}{1+(w-\mu)^{2}},
$$

where $\bar{\tau}$ is the unscaled steady wall shear, and the wavelength of the generalized Lam-Rott eigensolution (with respect to $s$ ) is given by

$$
\lambda_{\mathrm{LR}}(s)=\frac{2 \pi}{\operatorname{Im}(\mathrm{d} T / \mathrm{d} s)} .
$$

Due to the non-dimensionalization, $L(s)$ is independent of Strouhal number $S$, while $\lambda_{\mathrm{LR}}(s)$ does depend on $S$. The value of $\lambda_{\mathrm{LR}}(s)$ is obtained from (B 4), where the numerical rather than asymptotic value of $U_{0}^{\prime}(w)$ is used. The quantities $L(s)$ and $\lambda_{\mathrm{LR}}(s)$ are plotted in figure 9 for the case $\mu=0.9$ and for $S=0.1$ and $S=0.3$. For 


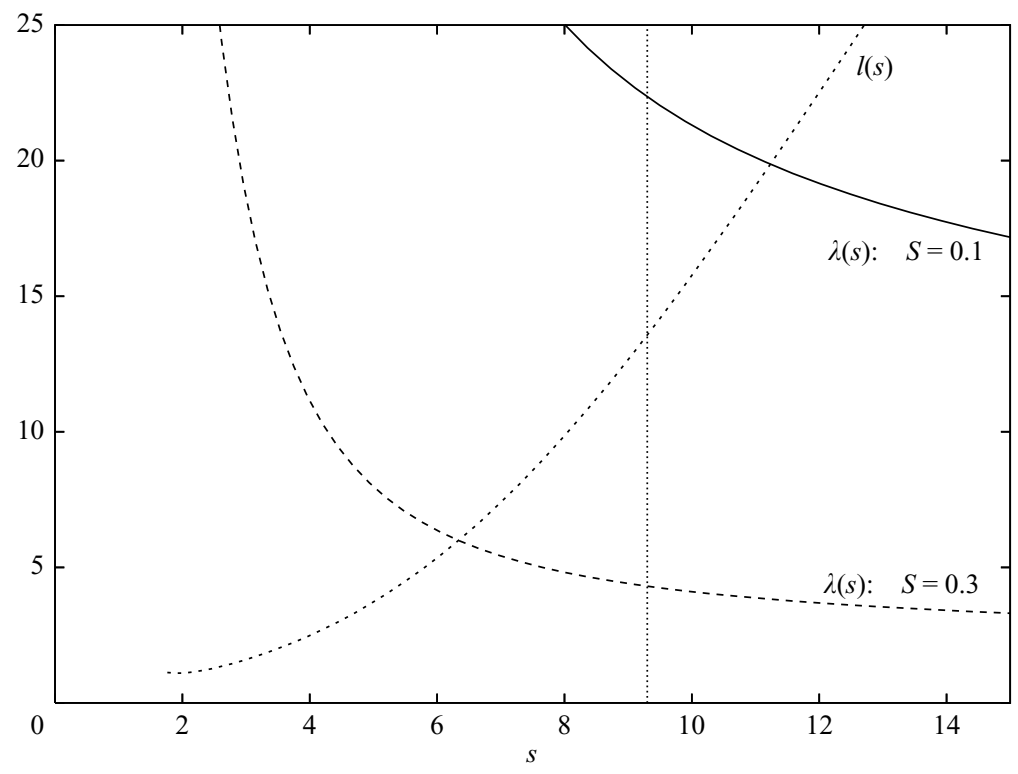

FiguRE 9. Comparison of the length scale of the development of the mean boundary layer $L(s)$ with the wavelength of the first eigensolution $\lambda_{\mathrm{LR}}(s)$ for $\mu=0.9$ and Strouhal numbers $S=0.1$ and $S=0.3$. The point of minimum wall shear, $s=9.4$ is also marked.

this value of $\mu$, the position of minimum wall shear occurs at $s \approx 9.4$. Note that $L(s)$ increases monotonically with $s$. There is no indication of shorter-scale behaviour near the point of minimum wall shear, showing that $\mu=0.9$ is quite far from the marginal separation regime. We would expect the receptivity to be concentrated in the region where $L(s)$ is less than the wavelength $\lambda_{\mathrm{LR}}(s)$ of the unsteady disturbance. From figure 9, it is clear that for $S=0.3$ the region of receptivity is located upstream of the region of low wall shear. For the case $S=0.1$, there may be some contribution from the region of low wall shear, but there is no reason to believe that there is a concentration of receptivity in this region. Thus, figure 9 provides further evidence that the region of low wall shear does not make an important contribution to the transfer of energy from the free-stream disturbance to the precursor of the instability wave, for the conditions considered here.

\section{Conclusions}

The results presented in the previous section lead to a number of general conclusions. It is clear that modest levels of aerodynamic loading in the leadingedge region cause a decrease in the receptivity level for the boundary layer on the upper surface of the airfoil, and an increase in receptivity for the lower surface. The effects are more pronounced at higher values of the Strouhal number, where the region of receptivity is concentrated nearer the stagnation point. Thus, it seems reasonable to suppose that the effects are related to the behaviour of the mean-flow pressure gradient near the nose of the airfoil. For the upper surface, the introduction of aerodynamic loading leads to a movement of the stagnation point toward the lower surface, and an increased favourable pressure gradient in the region between the stagnation point $(w=0)$ and the leading edge $(w=\mu)$. The decrease in receptivity level associated with these effects is consistent with the results obtained in HK1 
for the case $\mu=0$. The decrease in receptivity level on the upper surface in the presence of modest aerodynamic loading is of significance for applications such as laminar flow design. The subsequent rise in receptivity for larger values of $\mu$ also has important practical implications. For the lower surface, an argument that the increase in receptivity with modest aerodynamic loading is related to the pressure gradient near the stagnation point appears more tenuous, since the changes in the pressure gradient near the stagnation point are not pronounced. However, from a practical standpoint, receptivity levels for the lower surface are usually of less interest, since the pressure gradient on the lower surface is typically favourable and the instability waves have much smaller growth rates.

It must also be kept in mind that, for leading-edge receptivity, quantities such as the instability wave amplitude at the neutral stability point depend crucially on a combination of receptivity and stability properties. Furthermore, the instability wave depends linearly on the receptivity coefficient, while it depends exponentially on the instability growth rate. There can be a subtle interplay between these, since a small change in growth/decay rate can counteract a significant change in receptivity level. In fact, for the leading-edge receptivity mechanism, even the issue of the most appropriate definition of receptivity level is not straightforward. Experimental and computational researchers often favour a definition based on the lower branch amplitude. This is certainly of practical utility and has advantages with respect to measurements, but it does not distinguish between the receptivity and stability aspects of the disturbance development. The definition we have adopted has the advantage of separating the receptivity aspects from stability considerations, but of course the results must be combined with a stability calculation in order to predict amplitudes in the Orr-Sommerfeld region farther downstream.

This work was supported by NASA Langley Research Center under grant NAG1-1135 and Air Force Office of Scientific Research under grant F49620-94-1-0206.

\section{Appendix A. Asymptotic form of base flow far downstream}

The functions $p_{i}(\eta)$ in (3.12) are determined by

$$
\mathscr{L}_{i}\left(p_{i}\right)=d_{i},
$$

where

$$
\left.\begin{array}{l}
\mathscr{L}_{1}(p) \equiv p^{\prime \prime \prime}+F p^{\prime \prime}+F^{\prime} p^{\prime}, \\
\mathscr{L}_{2}(p) \equiv \mathscr{L}_{3}(p) \equiv p^{\prime \prime \prime}+F p^{\prime \prime}+2 F^{\prime} p^{\prime}-F^{\prime \prime} p, \\
\mathscr{L}_{4}(p) \equiv \mathscr{L}_{5}(p) \equiv p^{\prime \prime \prime}+F p^{\prime \prime}+3 F^{\prime} p^{\prime}-2 F^{\prime \prime} p,
\end{array}\right\}
$$

and

$$
\left.\begin{array}{rl}
d_{1} & =\mu\left[1-F^{\prime 2}\right], \\
d_{2} & =0, \\
d_{3} & =\left[F^{\prime 2}-1-2\left(p_{2} F^{\prime \prime}-p_{2}^{\prime} F^{\prime}\right)\right]-\mu^{2}\left[F^{\prime 2}-1+2 F^{\prime} p_{1}^{\prime}+p_{1}^{\prime 2}\right], \\
d_{4} & =\mu\left[p_{1}^{\prime \prime} p_{2}-3 p_{1}^{\prime} p_{2}^{\prime}-2 F^{\prime} p_{2}^{\prime}\right], \\
d_{5} & =\mu\left[p_{1}^{\prime \prime} p_{3}-3 p_{1}^{\prime} p_{3}^{\prime}-2 F^{\prime} p_{3}^{\prime}+2\left(F^{\prime} p_{4}^{\prime}-F^{\prime \prime} p_{4}\right)+2\left(p_{1}^{\prime} p_{2}^{\prime}-p_{1}^{\prime \prime} p_{2}\right)+2 F^{\prime} p_{1}^{\prime}\right. \\
& \left.\quad-3\left(1-F^{\prime 2}\right)\right]-\mu^{3}\left[F^{\prime 2}-1+2 F^{\prime} p_{1}^{\prime}+p_{1}^{\prime 2}\right] .
\end{array}\right\}
$$

These equations must be solved subject to the boundary conditions $p_{i}(0)=p_{i}^{\prime}(0)=0$, and the matching condition $p_{i}^{\prime} \rightarrow 0$ exponentially as $\eta \rightarrow \infty$. The latter condition 
is necessary to ensure that vorticity decays exponentially at the outer edge of the boundary layer.

The function $p_{1}(\eta)$ is the response of the mean boundary-layer profile to the $O\left(w^{-1}\right)$ term of the mean pressure gradient (3.11). An exact solution for $p_{1}(\eta)$ can be obtained in terms of the Blasius function $F(\eta)$; since the expression is cumbersome and only the wall shear stress $p_{1}^{\prime \prime}(0)$ appears in our final results, $p_{1}(\eta)$ is not presented here.

The second term in the mean pressure gradient is proportional to $w^{-2}$. However, if the series (3.12) for $\phi$ were assumed to proceed only in inverse powers of $w$, the solution that would be obtained for the $O\left(w^{-2}\right)$ term would not exhibit exponential decay of vorticity at the outer edge of the boundary layer. Exponential decay of vorticity is restored by including a term of $O\left(w^{-2} \log w^{2}\right)$ in (3.12), as discussed by Van Dyke (1964) for the symmetric flow case $(\mu=0)$.

The equation governing $p_{2}(\eta)$ is homogeneous but admits an eigensolution, $p_{2}=A_{1}\left(\eta F^{\prime}-F\right)$. The coefficient $A_{1}$ is then determined by a secularity condition on the differential equation $\mathscr{L}_{3}\left(p_{3}\right)=d_{3}$ governing the $O\left(w^{-2}\right)$ term. Multiplying both sides of this equation by $F$ and integrating, after some manipulation we obtain

$$
\left(1-\mu^{2}\right)\left\{\log \left(\frac{\gamma}{F_{0}^{\prime \prime}}\right)-\frac{1}{4}\right\}+A_{1}-\mu^{2} \int_{0}^{\infty} F\left(2 F^{\prime} p_{1}^{\prime}+p_{1}^{\prime 2}\right) \mathrm{d} \eta=0,
$$

where $F_{0}^{\prime \prime} \equiv F^{\prime \prime}(0)=0.4696$ and $\gamma=0.33054$, a constant appearing in the large- $\eta$ asymptotic form of $F(\eta)$. Thus, setting $A_{1}=\bar{A}_{1}+\mu^{2} \widetilde{A}_{1}$, the numerical values of these constants are found to be

$$
\bar{A}_{1}=0.60115, \quad \widetilde{A}_{1}=-2.1656 .
$$

Next consider the solution for $p_{3}(\eta)$. The operator $\mathscr{L}_{3}$ is identical to $\mathscr{L}_{2}$, so that $p_{3}(\eta)$ also contains an eigensolution $B_{1}\left(\eta F^{\prime}-F\right)$. However, in this case the function $w^{-2}\left(\eta F^{\prime}-F\right)$ is an eigensolution of the partial-differential perturbation equation (in fact, it is the first eigensolution of Libby \& Fox (1963)). Therefore, the coefficient $B_{1}(\mu)$ remains undetermined in the large- $w$ analysis. In $\S 5, B_{1}(\mu)$ is determined by comparing the large- $w$ asymptotic expression for the wall shear with results obtained by numerical integration of the mean flow equations starting from the stagnation point $w=0$.

The ordinary differential equation governing $p_{3}$ is inhomogeneous, so that a particular solution is required. The need for numerical solution for each value of $\mu$ can be avoided by noting the form of the dependence on $\mu$ of the inhomogeneous term $d_{3}$. Similar remarks apply for $p_{4}$ and $p_{5}$. Thus we set

$$
p_{3}=B_{1}\left(\eta F^{\prime}-F\right)+\bar{p}_{3}+\mu^{2} \tilde{p}_{3}, \quad p_{4}=\mu A_{1} \bar{p}_{4}, \quad p_{5}=\mu\left[B_{1} \bar{p}_{4}+\bar{p}_{5}\right]+\mu^{3} \tilde{p}_{5},
$$

where the functions $\bar{p}_{3}, \tilde{p}_{3}, \bar{p}_{4}, \bar{p}_{5}$ and $\tilde{p}_{5}$ satisfy third-order ordinary differential equations which are independent of $\mu$. Since $\mathscr{L}_{3}$ admits an eigensolution, we ensure uniqueness of $\bar{p}_{3}$ and $\tilde{p}_{3}$ by enforcing the additional conditions $\bar{p}_{3}^{\prime \prime}(0)=\tilde{p}_{3}^{\prime \prime}(0)=0$. The solutions for $\bar{p}_{3}, \tilde{p}_{3}, \bar{p}_{4}, \bar{p}_{5}$ and $\tilde{p}_{5}$ are obtained by numerical integration, with $\bar{p}_{4}^{\prime \prime}(0)$, $\bar{p}_{5}^{\prime \prime}(0)$ and $\tilde{p}_{5}^{\prime \prime}(0)$ determined by shooting.

Finally, the wall shear, $U_{0}^{\prime}(w) \equiv \phi_{\eta \eta}(w, 0)$, which appears in (3.13) has the asymptotic expansion

$$
\begin{aligned}
U_{0}^{\prime}(w)=F_{0}^{\prime \prime}\left(1+\mu \frac{\bar{l}_{0}}{w}+A_{1} \frac{\log w^{2}}{w^{2}}\right. & +\frac{B_{1}}{w^{2}}+\mu A_{1} \bar{j}_{0} \frac{\log w^{2}}{w^{3}} \\
& \left.+\mu \frac{B_{1} \bar{j}_{0}+\bar{k}_{0}+\mu^{2} \bar{l}_{0}}{w^{3}}+O\left(w^{-\gamma_{2}}\right)\right),
\end{aligned}
$$


where the numerical constants are calculated to be

$$
\bar{\imath}_{0}=-3.591, \quad \bar{j}_{0}=0.814, \quad \bar{k}_{0}=8.230, \quad \bar{l}_{0}=-3.235 .
$$

\section{Appendix B. Asymptotic form of disturbance far downstream}

In this Appendix, we develop expressions for the large- $\xi$ asymptotic eigenfunctions of the LUBLE. For algebraic simplicity, the analysis is carried out in terms of large $w$ (see (3.3)) with $S$ assumed of $O(1)$. Guided by the flat-plate results of Ackerburg \& Phillips (1972), we anticipate that the asymptotic eigensolutions have a two-layer structure, with an inner layer of thickness $\eta=O\left(S^{-1 / 2} w^{-1}\right)$, and a main layer of the same thickness as the mean boundary layer, $\eta=O(1)$.

\section{B.1. Inner layer}

Introducing an inner variable $m=S^{1 / 2} w \eta$, the homogenous form of the LUBLE becomes

$$
\begin{aligned}
& \psi_{m m m}+\mathrm{i}\left(1-\frac{2 \mu}{w}+\frac{1+\mu^{2}}{w^{2}}\right) \psi_{m}-\frac{m F_{0}^{\prime \prime}}{2 S^{3 / 2} w^{3}}\left(m \psi_{m m}-2 \psi_{m}\right)+o\left(w^{-3}\right) \\
& =\frac{U_{0}^{\prime}(w)}{S^{3 / 2} w^{2}}\left(m \psi_{m w}-\psi_{w}\right)+\frac{m U_{0}^{\prime \prime}(w)}{2 S^{2} w^{3}}\left(m \psi_{m w}-2 \psi_{w}\right)-\frac{m^{3} F_{0}^{\prime \prime 2}}{24 S^{3} w^{5}}\left(m \psi_{m w}-4 \psi_{w}\right),
\end{aligned}
$$

where $U_{0}^{\prime}(w)$ is given by (A 7) and $U_{0}^{\prime \prime}(w)=-\beta(w)$ is given by (3.11). The regular perturbation solution to (B 1), for large $w$, leads to solutions related to the particular solution $\psi_{p}$. Thus, the asymptotic eigensolutions must arise as a balance between the highest $m$-derivative and the terms involving $w$-derivatives. It is then clear that the eigensolution must contain a factor $\exp (T(w))$ and it is for this reason that the higher-order terms containing streamwise derivatives have been retained in (B 1).

The subsequent analysis is simplified somewhat by absorbing some of the effects of geometry and aerodynamic loading into a new inner variable,

$$
M=\left(1-\mu w^{-1}+\frac{1}{2} w^{-2}+\frac{1}{2} \mu w^{-3}\right) m .
$$

Next, setting

$$
\psi(w, M)=\exp (T(w)) \theta(w, M)
$$

in (B1) and rearranging in order to separate the functions $T$ and $\theta$, we obtain an expression for the derivative of $T$,

$$
\frac{\mathrm{d} T}{\mathrm{~d} w}=-\lambda \frac{S^{3 / 2}}{U_{0}^{\prime}(w)} w^{2}\left(1-\mu w^{-1}+\frac{1}{2} w^{-2}+\frac{1}{2} \mu w^{-3}\right)^{3},
$$

and a differential equation for the mode-shape function,

$$
\theta_{M M M}+\mathrm{i} \theta_{M}+\lambda\left(M \theta_{M}-\theta\right)=w^{-2} \bar{R}_{2}+w^{-3} \bar{R}_{3}+o\left(w^{-3}\right),
$$

where $\lambda$ is the 'separation constant' and the remainder terms on the right-hand side of (B 5) are given by

$$
\begin{aligned}
& \bar{R}_{2}=-\frac{\lambda \mu}{2 F_{0}^{\prime \prime} S^{1 / 2}} M\left(M \theta_{M}-2 \theta\right) \\
& \left.\begin{array}{rl}
\bar{R}_{3}= & \frac{F_{0}^{\prime \prime}}{S^{3 / 2}}\left(\frac{1}{2} M\left(M \theta_{M M}-2 \theta_{M}\right)+w\left(M \theta_{M w}-\theta_{w}\right)-\frac{1}{24} \lambda M^{3}\left(M \theta_{M}-4 \theta\right)\right. \\
& \left.+\frac{\lambda\left[1-\mu^{2}\left(2-\bar{\imath}_{0}\right)\right]}{2 F_{0}^{\prime \prime 2}} S M\left(M \theta_{M}-2 \theta\right)\right) .
\end{array}\right\}
\end{aligned}
$$


The mode-shape function satisfies homogeneous boundary conditions at the wall,

$$
\theta=\theta_{M}=0 \quad \text { at } \quad M=0,
$$

while matching to the main layer requires suppression of any terms exhibiting exponential growth as $M \rightarrow \infty$.

If the small remainder terms on the right-hand side of (B 5) were not present, (B 5) and (B 7) would define an eigenvalue problem for the mode-shape function $\theta(M)$ and the eigenvalue $\lambda$. Due to the presence of the remainder terms, the eigenvalue $\lambda$ exhibits a slow dependence on $w$ for $w \gg 1$, and the mode-shape function also exhibits a slow dependence on $w$. Thus we set

$$
\begin{aligned}
\lambda & =\lambda_{0}+\lambda_{2} w^{-2}+\lambda_{3} w^{-3}+o\left(w^{-3}\right), \\
\theta(w, M) & =\theta_{0}(M)+\theta_{2}(M) w^{-2}+\theta_{3}(M) w^{-3}+o\left(w^{-3}\right) .
\end{aligned}
$$

Substituting these expansions into (B 5) and (B 7), the leading-order terms produce an eigenvalue problem involving $\theta_{0}(M)$ and $\lambda_{0}$, while higher-order terms provide the equations governing $\theta_{j}(M)$ and $\lambda_{j}, j=2,3, \ldots$.

The analysis is simplified by differentiating (B 5) once with respect to $M$, then introducing a new independent variable

$$
z=\left(-\mathrm{i} \lambda_{0} M+1\right) z_{0} \quad \text { where } \quad z_{0}^{3} \lambda_{0}^{2}=\mathrm{i},
$$

and changing the dependent variable to

$$
f_{j}=\frac{\mathrm{d}^{2} \theta_{j}}{\mathrm{~d} z^{2}}, \quad j=0,2,3, \ldots
$$

The governing equations at leading order then take the form

$$
f_{0}^{\prime \prime}(z)-z f_{0}(z)=0, \quad f_{0}^{\prime}\left(z_{0}\right)=0,
$$

where the boundary condition at the wall $z=z_{0}$ follows from (B 5) and (B 7), and exponential growth of $f_{0}(z)$ as $z \rightarrow \infty$ must be suppressed. The solution is

$$
f_{0}(z)=D \operatorname{Ai}(z), \quad \lambda_{0}=\rho^{-3 / 2} \mathrm{e}^{-\mathrm{i} \pi / 4}, \quad z_{0}=-\rho,
$$

where $D$ is an arbitrary constant and $\rho=\rho_{i}$ is one of the infinite sequence of positive roots of $\mathrm{Ai}^{\prime}(-\rho)=0$. The first eigenfunction, corresponding to $\rho=\rho_{1}$, matches onto the TS wave of the Orr-Sommerfeld region where $S^{1 / 2} w=O\left(\epsilon^{-1}\right)$. For convenience, the subscript ' $i$ ' denoting the mode index of the asymptotic eigensolution is not displayed explicitly in the remainder of this Appendix.

The equations for the higher-order corrections to the mode-shape function now take the form

$$
f_{j}^{\prime \prime}(z)-z f_{j}(z)=D G_{j}(z), \quad j=2,3, \ldots,
$$

where

$$
\begin{aligned}
G_{2}(z)= & \frac{\mathrm{e}^{-\mathrm{i} \pi / 4} \rho^{1 / 2} \mu}{F_{0}^{\prime \prime} S^{1 / 2}}\left[I_{1}(z)-\frac{1}{2}\left(z-z_{0}\right)^{2} \operatorname{Ai}(z)\right]-\lambda_{2} \mathrm{e}^{\mathrm{i} \pi / 4} \rho^{3 / 2}\left(z-z_{0}\right) \operatorname{Ai}(z), \\
G_{3}(z)= & \frac{\mathrm{e}^{-\mathrm{i} 3 \pi / 4} F_{0}^{\prime \prime} \rho^{3 / 2}}{S^{3 / 2}}\left[I_{0}(z)-\frac{1}{2}\left(z-z_{0}\right)^{2}\left[\operatorname{Ai}^{\prime}(z)+I_{1}(z)\right]+\frac{1}{24}\left(z-z_{0}\right)^{4} \operatorname{Ai}(z)\right] \\
& -\frac{\mathrm{e}^{-\mathrm{i} \pi / 4} \rho^{1 / 2}}{F_{0}^{\prime \prime} S^{1 / 2}}\left[1-\mu^{2}\left(2-\bar{\imath}_{0}\right)\right]\left[I_{1}(z)-\frac{1}{2}\left(z-z_{0}\right)^{2} \operatorname{Ai}(z)\right] \\
& -\lambda_{3} \mathrm{e}^{\mathrm{i} \pi / 4} \rho^{3 / 2}\left(z-z_{0}\right) \operatorname{Ai}(z)
\end{aligned}
$$


with $I_{j}(z)=\int_{z p}^{z}\left(z-z^{\prime}\right)^{j} \operatorname{Ai}\left(z^{\prime}\right) \mathrm{d} z^{\prime}$. The functions $f_{2}(z)$ and $f_{3}(z)$ satisfy the same boundary conditions as $f_{0}(z)$. Thus, in order for the solutions $f_{2}(z)$ and $f_{3}(z)$ to exist, the solvability conditions $\int_{z_{0}}^{\infty} \operatorname{Ai}(z) G_{j}(z) \mathrm{d} z=0, j=2,3$ must be satisfied. These lead to

$$
\begin{aligned}
& \lambda_{2}=\frac{\mathrm{i} \mu}{2 F_{0}^{\prime \prime} S^{1 / 2} \rho} \frac{J_{2}-2 \mathrm{Ai}^{2}\left(z_{0}\right)}{J_{1}}=\frac{\mathrm{i} \mu\left[8 \rho^{3}-27\right]}{20 F_{0}^{\prime \prime} S^{1 / 2} \rho^{3}}, \\
& \lambda_{3}=-\frac{2 F_{0}^{\prime \prime}}{S^{3 / 2}}\left(\tau^{(0)}+\mathrm{i} S \tau^{(1)}\left[1-\mu^{2}\left(2-\bar{\imath}_{0}\right)\right]\right),
\end{aligned}
$$

where

$$
\tau^{(0)}=\left[\frac{1}{48} \frac{J_{4}}{J_{1}}-\frac{3}{4}\right]=\frac{16 \rho^{3}-889}{1260}, \quad \tau^{(1)}=\frac{\left[2 \mathrm{Ai}^{2}\left(z_{0}\right)-J_{2}\right]}{4 F_{0}^{\prime \prime 2} \rho J_{1}}=\frac{8 \rho^{3}-27}{40 F_{0}^{\prime \prime 2} \rho^{3}} .
$$

Here $J_{j}=\int_{z_{0}}^{\infty}\left[z-z_{0}\right]^{j} \mathrm{Ai}^{2}(z) \mathrm{d} z$; recursion relationships for these quantities are given in the Appendix of HK1.

With $\lambda$ determined, we now integrate (B 4) to obtain the exponent $T(w)$, which we write in the form

where

$$
T(w)=T^{(0)}(w)+2 \tau \log w+T^{(1)}(w),
$$

$$
\begin{aligned}
\tau & =\tau^{(0)}+\mathrm{i} S \tau^{(1)}\left[1+\mu^{2}\left(2 \bar{\imath}_{0}+1\right)\right], \\
T^{(0)} & =\frac{\mathrm{e}^{\mathrm{i} 3 \pi / 4} S^{3 / 2}}{F_{0}^{\prime \prime} \rho^{3 / 2}}\left[\frac{1}{3} w^{3}+a_{1} w^{2}+a_{2} w \log w+a_{3} w+a_{4} \log ^{2} w+a_{5} \log w\right], \\
T^{(1)} & =b_{1} w^{-0.774}+w^{-1}\left(b_{2} \log ^{2} w+b_{3} \log w+b_{4}\right)+o\left(w^{-1}\right) .
\end{aligned}
$$

For large $w$, the terms in $T^{(0)}$ are large and hence must be included in the leadingorder form of the eigensolution $\psi$. The term involving $\tau$ provides the $O(1)$ algebraic dependence of $\psi$ on $w$. The terms in $T^{(1)}$ are small for $w \gg 1$; thus $\exp \left(T^{(1)}\right)$ could be expressed as higher-order corrections to the algebraic dependence of $\psi$ on $w$. In principle, the coefficients $b_{j}$ could be determined by retaining higher-order terms in the asymptotic expansions, though this is unnecessary for present purposes.

The large number of terms retained in the various asymptotic expansions were required in order to determine the coefficients $a_{j}$,

$$
\left.\begin{array}{l}
a_{1}=-\frac{1}{2} \mu\left(\bar{\imath}_{0}+3\right), \\
a_{2}=-2 A_{1}, \\
a_{3}=\frac{3}{2}+2 A_{1}-B_{1}+\mu^{2}\left(\bar{\imath}_{0}^{2}+3 \bar{\imath}_{0}+3\right)-2 \mu \rho^{3 / 2} S^{-1 / 2} F_{0}^{\prime \prime} \tau^{(1)} \mathrm{e}^{-\mathrm{i} \pi / 4}, \\
a_{4}=A_{1} \mu\left(2 \bar{\imath}_{0}-\bar{h}_{0}+3\right), \\
a_{5}=-\mu^{3}\left(\bar{\imath}_{0}^{3}+3 \bar{\imath}_{0}^{2}+3 \bar{l}_{0}+1+\bar{k}_{0}\right)+\mu\left[B_{1}\left(3+2 \bar{\imath}_{0}-\bar{h}_{0}\right)-\frac{3}{2}\left(1+\bar{\imath}_{0}\right)-\bar{j}_{0}\right] .
\end{array}\right\}
$$

Note that for $\mu>0, a_{1}$ is positive and so at leading order, the disturbances decay faster on the upper surface than for the flat-plate case.

Returning to (B 11) and (B 13), the leading-order mode shape can be obtained by integrating twice to give

$$
\theta_{0}(M)=F_{0}^{\prime \prime}\left(\frac{S}{2}\right)^{\tau} \frac{\int_{0}^{M}(M-\widetilde{M}) \operatorname{Ai}\left(\mathrm{e}^{\mathrm{i} \pi / 4} \rho^{-1 / 2} \widetilde{M}-\rho\right) \mathrm{d} \widetilde{M}}{\int_{0}^{\infty} \operatorname{Ai}\left(\mathrm{e}^{\mathrm{i} \pi / 4} \rho^{-1 / 2} \widetilde{M}-\rho\right) \mathrm{d} \widetilde{M}},
$$


where the arbitrary constant $D$ that multiplies the asymptotic eigenfunction has been chosen so that in the limiting case $\mu \rightarrow 0$ it agrees with the result for the zero-angle-of-attack case of HK1.

\section{B.2. Main layer and matching}

Turning now to the main layer, where $\eta=O(1)$, we write the asymptotic eigenfunction in the form

$$
\psi=w^{2 \tau+1} \exp \left(T^{(0)}(w)+T^{(1)}(w)\right) g(w, \eta) .
$$

The governing equation becomes

$$
\phi_{\eta} g_{\eta}-\phi_{\eta \eta} g=\frac{\mathrm{e}^{-\mathrm{i} \pi / 4} F_{0}^{\prime \prime} \rho^{3 / 2}}{S^{1 / 2} w} g_{\eta}+O\left(w^{-2}\right) .
$$

Thus

$$
g=A(w)\left[\phi_{\eta}-\frac{\mathrm{e}^{-\mathrm{i} \pi / 4} F_{0}^{\prime \prime} \rho^{3 / 2}}{S^{1 / 2} w}+O\left(w^{-2}\right)\right]
$$

where $A(w)$ is determined by matching to the inner layer. This is completed most easily by matching $\psi_{\eta}$, to give

$$
A(w)=S^{1 / 2}\left(\frac{S}{2}\right)^{\tau}\left(1-\frac{\mu\left(1+\bar{\iota}_{0}\right)}{w}+O\left(w^{-2}\right)\right) .
$$

\section{REFERENCES}

ACKerberg, R. C. \& Phillips, J. H. 1972 The unsteady laminar boundary layer on a semi-infinite flat plate due to small fluctuations in the magnitude of the free-stream velocity. J. Fluid Mech. 51, 137-157.

Brown, S. N. \& Stewartson, K. 1973 On the propagation of disturbances in a laminar boundary layer. Proc. Camb. Phil. Soc. 73, 493-514.

Cheng, H. K. \& Rott, N. 1954 Generalizations of the inversion formula of thin airfoil theory. J. Rat. Mech. Anal. 3, 357-382.

Choudhari, M. \& Kerschen, E. J. 1990 Instability wave patterns generated by interaction of sound waves with three-dimensional wall suction or roughness AIAA Paper 90-0119.

Erturk, E. \& Corke, T. C. 2001 Boundary layer leading-edge receptivity to sound at incidence angles. J. Fluid Mech. 444, 383-407.

Goldstein, M. E. 1983 The evolution of Tollmien-Schlichting waves near a leading edge. J. Fluid Mech. 127, 59-81.

Goldstein, M. E. 1985 Scattering of acoustic waves into Tollmien-Schlichting waves by small streamwise variations in surface geometry. J. Fluid Mech. 154, 509-529.

Goldstein, M. E. \& Hultgren, L. S. 1989 Boundary-layer receptivity to long-wave free-stream disturbances. Annu. Rev. Fluid Mech. 21, 137-166.

Goldstein, M. E., Leib, S. J. \& Cowley, S. J. 1987 Generation of Tollmien-Schlichting waves on interactive marginally separated flows. J. Fluid Mech. 181, 485-517.

Goldstein, M. E., Sockol, P. M. \& Sanz, J. 1983 The evolution of Tollmien-Schlichting waves near a leading edge. Part 2. Numerical determination of amplitudes. J. Fluid Mech. 129, 443 453.

HAdDad, O. M. \& CoRKe, T. C. 1998 Boundary layer receptivity to free-stream sound on parabolic bodies. J. Fluid Mech. 368, 1-26.

Hammerton, P. W. \& Kerschen, E. J. 1996 Boundary-layer receptivity for a parabolic leading edge. J. Fluid Mech. 310, 243-267 (referred to herein as HK1).

Hammerton, P. W. \& Kerschen, E. J. 1997 Boundary-layer receptivity for a parabolic leading edge. Part 2. The small-Strouhal number limit. J. Fluid Mech. 353, 205-220.

HeINRICH, R. A. E. \& KersChen, E. J. 1989 Leading-edge boundary layer receptivity to various free-stream disturbance structures. Z. Angew. Math. Mech. 69 6, T596-598. 
Keller, H. B. \& Cebeci, T. 1970 Accurate numerical methods for boundary layer flows-I. Two-dimensional laminar flows. In Proc. Second Intl. Conf. on Numerical Methods in Fluid Mechanics (ed. M. Holt), pp. 92-100. Springer.

Kerschen, E. J. 1990 Boundary layer receptivity theory. Appl. Mech. Rev. 43, 152-157.

Kozlov, V. V. \& Ryzhov, O. S. 1990 Receptivity of boundary layers: asymptotic theory and experiment. Proc. R. Soc. Lond. A 429, 341-373.

Lam, S. H. \& RotT, N. 1960 Theory of linearized time-dependent boundary layers. Cornell Univ. Grad. School of Aero. Engng Rep. AFOSR TN-60-1100.

Lam, S. H. \& RotT, N. 1993 Eigen-functions of linearized unsteady boundary layer equations. Trans. ASME: J. Fluids Engng 115, 597-602.

LibBy, P. A. \& Fox, H. 1963 Some perturbation solutions in laminar boundary layer theory. Part 1: The momentum equation. J. Fluid Mech. 17, 433-449.

Morkovin, M. V. 1969 Critical evaluation of transition from laminar to turbulent shear layers with emphasis on hypersonically traveling bodies. Air Force Flight Dyn Lab., Wright-Patterson $A F B$, Ohio. AF FDL-TR-68-149.

ReEd, H. L. \& LytTLE, I. 1998 DN S of leading edge receptivity to sound. AIAA Paper 98-2644.

Ruban, A. I. 1982 Asymptotic theory of short separation regions on the leading edge of a slender airfoil. Fluid Dyn. 17, 33-41 (Engl. Transl.).

Ruban, A. I. 1984 On the generation of Tollmien-Schlichting waves by sound. Izv. Akad. Nauk SSSR, Mech. Zhidk. \& Gaza. 5, 44-52.

SARIC, W. S., Reed, H. L. \& Kerschen, E. J. 2002 Boundary-layer receptivity to freestream disturbances. Annu. Rev. Fluid Mech. 34, 291-319.

Saric, W. S., Wei, W., Rasmussen, B. K. \& Krutckoff, T. K. 1995 Experiments on leading edge receptivity to sound. AIAA Paper 95-2253.

SARIC, W. S. \& White, E. B. 1998 The influence of high amplitude noise on boundary layer transition to turbulence. AIAA Paper 98-2645.

Stewartson, K., Smith, F. T. \& Kaups, K. 1982 Marginal separation. Stud. Appl. Maths 67, 45-61.

VAN DyKe, M. D. 1964 Higher approximations in boundary layer theory. Part 3. Parabola in uniform stream. J. Fluid Mech. 19, 145-159. 